\title{
FCER1G positively relates to macrophage infiltration in clear cell renal cell carcinoma and contributes to unfavorable prognosis by regulating tumor immunity
}

Keqin Dong ${ }^{1 \dagger}$, Wenjin Chen ${ }^{1 \dagger}$, Xiuwu Pan ${ }^{1 \dagger}$, Hongru Wang ${ }^{1 \dagger}$, Ye Sun ${ }^{2}$, Cheng Qian ${ }^{2}$, Weijie Chen ${ }^{1}$, Chao Wang ${ }^{3^{*}}$, Fu Yang ${ }^{4^{*}}$ and Xingang Cui ${ }^{*}$

\begin{abstract}
Background: Tumor-associated macrophages (TAMs) are closely related to unfavorable prognosis of patients with clear cell renal cell carcinoma (ccRCC). However, the important molecules in the interaction between ccRCC and TAMs are unclear.

Methods: TCGA-KIRC gene expression data of tumor tissues and normal tissues adjacent to tumor were compared to identify differentially expressed genes in ccRCC. TAMs related genes were discovered by analyzing the correlation between these differentially expressed genes and common macrophage biomarkers. Gene set enrichment analysis was performed to predict functions of TAMs related gene. The findings were further validated using RNA sequencing data obtained from the CheckMate 025 study and immunohistochemical analysis of samples from 350 patients with ccRCC. Kaplan-Meier survival curve, Cox regression analysis and Harrell's concordance index analysis were used to determine the prognostic significance.

Results: In this study, we applied bioinformatic analysis to explore TAMs related differentially expressed genes in CCRCC and identified 5 genes strongly correlated with all selected macrophage biomarkers: STAC3, LGALS9, TREM2, FCERIG, and PILRA. Among them, FCERIG was abundantly expressed in tumor tissues and showed prognostic importance in patients with ccRCC who received treatment with Nivolumab; however, it did not exhibit prognostic value in those treated with Everolimus. We also discovered that high expression levels of FCER1G are related to T cell suppression. Moreover, combination of FCER1G and macrophage biomarker CD68 can improve the prognostic stratification
\end{abstract}

\footnotetext{
*Correspondence: superwang2012@aliyun.com; yangfusq@smmu.edu.cn;

cuixingang@smmu.edu.cn

${ }^{\dagger}$ Keqin Dong, Wenjin Chen, Xiuwu Pan and Hongru Wang contributed

equally to this work.

${ }^{1}$ Department of Urinary Surgery, Third Affiliated Hospital of Naval Medical University (Eastern Hepatobiliary Surgery Hospital), 700 North Moyu Road, 201805 Shanghai, China

${ }^{3}$ Department of Urinary Surgery, Gongli Hospital, Second Military Medical University (Naval Medical University), 219 Miaopu Road, 200135 Shanghai, China

${ }^{4}$ The Department of Medical Genetics, Naval Medical University, 800

Xiangyin Road, 200433 Shanghai, China

Full list of author information is available at the end of the article
}

(c) The Author(s) 2022. Open Access This article is licensed under a Creative Commons Attribution 4.0 International License, which permits use, sharing, adaptation, distribution and reproduction in any medium or format, as long as you give appropriate credit to the original author(s) and the source, provide a link to the Creative Commons licence, and indicate if changes were made. The images or other third party material in this article are included in the article's Creative Commons licence, unless indicated otherwise in a credit line to the material. If material is not included in the article's Creative Commons licence and your intended use is not permitted by statutory regulation or exceeds the permitted use, you will need to obtain permission directly from the copyright holder. To view a copy of this licence, visit http://creativecommons.org/licenses/by/4.0/. The Creative Commons Public Domain Dedication waiver (http://creativeco mmons.org/publicdomain/zero/1.0/) applies to the data made available in this article, unless otherwise stated in a credit line to the data. 
of patients with cCRCC from TCGA-KIRC. Based on the immunohistochemical analysis of samples from patients with CCRCC, we further validated that FCER1G and CD68 are both highly expressed in tumor tissue and correlate with each other. Higher expression of CD68 or FCER1G in cCRCC tissue indicates shorter overall survival and progression-free survival; patients with high expression of both CD68 and FCER1G have the worst outcome. Combining CD68 and FCER1G facilitates the screening of patients with a worse prognosis from the same TNM stage group.

Conclusions: High expression of FCER1G in CCRCC is closely related to TAMs infiltration and suppression of T cell activation and proliferation. Combining the expression levels of FCER1G and macrophage biomarker CD68 may be a promising postoperative prognostic index for patients with ccRCC.

Keywords: Clear cell renal cell carcinoma, Bioinformatic analysis, Tumor-associated macrophage, FCER1G, Prognosis, Tumor microenvironment

\section{Background}

The incidence of renal cell carcinoma (RCC) has been increasing as a result of the routine application of abdominal imaging. Clear cell renal cell carcinoma (ccRCC) is the most common pathological subtype of RCC, accounting for approximately $75 \%$ of all RCC cases and most cancer-related deaths [1]. Surgical resection is the standard treatment for localized ccRCC. Nevertheless, $25-40 \%$ of patients develop metastatic lesions even after radical treatment [2]. Following disease progression to an advanced state, the patients depend on tyrosine kinase inhibitors (TKIs) as first-line intervention; however, resistance to TKIs inevitably develops after standard treatment [3]. Therefore, an enhanced understanding of key regulatory molecules in ccRCC is urgently needed to more accurately estimate the long-term outcome of postoperative patients and develop more durable treatment options.

In recent years, the tumor immune microenvironment has attracted considerable research attention. Infiltration of immune-inhibitory cells is a hallmark of ccRCC that alters therapeutic effectiveness [4]. Clinical trials of immune checkpoint inhibitor (ICI) versus TKI treatment for ccRCC, such as CheckMate 214 [5], CheckMate 9ER [6], Keynote-426 [7], and IMmotion 151 [8] have shown pronounced clinical benefits. A recent update of the 4-year follow-up results of the CheckMate 214 study also showed durable efficacy [9], prompting researchers to reconsider the possibility of targeting tumor immunity as a long-term effective treatment for advanced ccRCC. However, a considerable proportion of patients showed limited reaction to ICI, while the underlying mechanism remains poorly understood [10].

Tumor-associated macrophages (TAMs) are key immune cell populations in ccRCC with great diversity in phenotype and functions [11]. They act through a variety of mechanisms to compromise the effects of treatment with TKIs and ICIs, including the promotion of angiogenesis [12], acceleration of tumor growth [13], and suppression of antitumor immunity [14]. We have previously reported that several molecules, such as SRYbox transcription factor 17 (SOX17) [15] and gankyrin [16], can regulate the function of TAMs to facilitate the progression of ccRCC. Hence, targeting TAMs is a potential option for developing treatments against ccRCC and obtaining a synergic effect from the utilization of TKIs and ICIs [17]. To determine the mechanism through which TAMs interact with ccRCC, we investigated whether other key molecules govern the biological function of TAMs in this disease. For this purpose, we used The Cancer Genome Atlas-kidney renal clear cell carcinoma (TCGA-KIRC) dataset to predict macrophagerelated molecules and validated the findings in a large cohort of patients from the clinical setting.

\section{Methods \\ Bioinformatics analysis}

The RNA sequencing (RNA-seq) count data of 538 tumors and 72 normal tissues adjacent to tumor (NATs) from TCGA-KIRC were downloaded from the online database using the $\mathrm{R}$ package 'TCGAbiolinks' [18]. Array Intensity correlation analysis was performed using the 'TCGAanalyze_Preprocessing' function to remove outliers (cut-off correlation score: 0.6). The 'TCGAtumor_purity' function was used to exclude unqualified tumor samples (tumor purity level $<60 \%$ ). The count data of TCGA-KIRC were further normalized using the GC-content method [19]. To obtain a more rigorous outcome, genes with expression levels $<25 \%$ of the average expression were excluded. Analysis of differentially expressed genes (DEGs) was performed using the R package 'Limma'; the expression data were transformed as $\log (\exp +1)$, while $\mid \log$ fold change $\mid>0.1$ and adjusted $P$-value $<1$ e-10 denoted significant differences in expression. Normalized bulk RNA-seq and clinical data of patients with ccRCC treated with either programmed cell death 1 (PD-1) blockade or mechanistic target of rapamycin kinase (MTOR) inhibition were obtained from the CheckMate 025 study [20]. To perform functional enrichment analysis, DEGs were loaded into clusterProfiler [21] 
for Gene Ontology and Kyoto Encyclopedia of Genes and Genomes enrichment analyses. Pathways with an adjusted $P$-value $<0.05$ were considered significantly enriched. Gene set enrichment analysis (GSEA) was conducted to detect significantly enriched gene sets. Only gene sets with false discovery rate and nominal $P$-values $<0.05$ were considered significantly enriched.

\section{Patients and specimens}

RCC tissue microarrays (TMAs) of 407 patients from Shanghai Changhai Hospital were included in this study; they involved two series. The first series (TMA-30) consisted of 29 patients with ccRCC (from our previous research) $[15,16]$, who underwent surgery from 2010 to 2015. Follow-up of all patients in TMA-30 via telephone interviews confirmed either disease progression (recurrence/metastasis) or cancer-related death. The TMA30 microarray included 10 pairs of tumors and NATs together with 19 cases of unpaired tumor tissue; two replicates were performed for each included sample. For another series (TMA-2020 NO.1-8) 378 patients with RCC who underwent surgery from 2016 to 2018 were indifferently included in eight TMAs. Of all included patients in TMA-2020 NO.1-8, 321 had ccRCC according to pathological examination. TMA-2020 NO.1-8 consists of 270 pairs of ccRCC tumors and NATs together with 50 cases of unpaired tumor tissue. We were able to contact 266 of the 321 patients with ccRCC for follow-up through telephone interviews. The clinical data (e.g., age, sex, TNM stage, and Fuhrman grade) of both series are summarized in Table 1. (Additional table files show this in more detail (see Additional file 1 and 2)). The primary outcomes were overall survival (OS) and progression-free survival (PFS). OS was defined as the duration of followup from surgery to the date of cancer-related death. PFS was defined as the duration of follow-up from surgery to the date of clinically diagnosed metastasis or recurrence of ccRCC. Samples for the IHC positive control were kindly donated by the Pathology Department of Shanghai Changhai Hospital. All experiments were approved by

Table 1 Characteristics of patients with clear cell renal cell carcinoma

\begin{tabular}{|c|c|c|c|}
\hline \multirow[t]{2}{*}{ Characteristics } & \multicolumn{2}{|c|}{ Cohort description } & \multirow{2}{*}{$\begin{array}{l}\text { Total } \\
(n=350)\end{array}$} \\
\hline & TMA-30 $(n=29)$ & TMA-2020 NO.1-8 $(n=321)$ & \\
\hline \multicolumn{4}{|l|}{ Age, years } \\
\hline Mean $\pm S D$ & $60.3 \pm 10.9$ & $57.0 \pm 11.9$ & $57.2 \pm 11.9$ \\
\hline Median & 63 & 55 & 56 \\
\hline Range & $39-80$ & $23-86$ & $23-86$ \\
\hline \multicolumn{4}{|l|}{ Sex, n (\%) } \\
\hline Male & $24(82.8)$ & $224(69.8)$ & $248(70.9)$ \\
\hline Female & $5(17.2)$ & $97(30.2)$ & $102(29.1)$ \\
\hline \multicolumn{4}{|c|}{ Fuhrman grade, n (\%) } \\
\hline $1-2$ & $18(62.1)$ & $261(81.3)$ & $279(79.7)$ \\
\hline $3-4$ & $11(37.9)$ & $60(18.7)$ & $71(20.3)$ \\
\hline \multicolumn{4}{|l|}{ TNM stage, n (\%) } \\
\hline$|-| \mid$ & $23(79.3)$ & $275(85.7)$ & $298(85.1)$ \\
\hline III-IV & $6(20.7)$ & $45(14.0)$ & $51(14.6)$ \\
\hline NA & 0 & $1(0.3)$ & $1(0.3)$ \\
\hline \multicolumn{4}{|l|}{ T category, n (\%) } \\
\hline T1a-T1b & $19(65.5)$ & $252(50.5)$ & $271(77.4)$ \\
\hline T2a-T4 & $10(34.5)$ & $68(49.2)$ & $78(22.3)$ \\
\hline NA & 0 & $1(0.3)$ & $1(0.3)$ \\
\hline \multicolumn{4}{|c|}{ Overall survival, n (\%) } \\
\hline- & $2(6.9)$ & $249(77.6)$ & $251(71.7)$ \\
\hline+ & $27(93.1)$ & $17(5.3)$ & $44(12.6)$ \\
\hline NA & 0 & $55(17.1)$ & $55(15.7)$ \\
\hline \multicolumn{4}{|c|}{ Progression-free survival, $\mathrm{n}(\%)$} \\
\hline- & $5(17.2)$ & $236(73.5)$ & $241(68.9)$ \\
\hline+ & $24(82.8)$ & $30(9.3)$ & $54(15.4)$ \\
\hline NA & 0 & $55(17.1)$ & $55(15.7)$ \\
\hline
\end{tabular}

NA not applicable; SD standard deviation; TMA tissue microarray 
the Scientific Research Review and Investigation Committee of the Third Affiliated Hospital of the Second Military Medical University (ID:EHBHKY2020-K-026, approval date: August $17^{\text {th }}, 2020$ ), and written informed consent was provided by all patients.

\section{IHC}

IHC assay was conducted as previously described [15]. Briefly, paraffin-embedded sections of ccRCC TMA were preprocessed with the antigen retrieval procedure. For the antibody against CD68, citric acid was used at a $\mathrm{pH}$ of 6.0. For the antibody against FCER1G, ethylene diamine tetraacetic acid was used at a $\mathrm{pH}$ of 9.0. Goat serum $(10 \%)$ was used to block nonspecific binding. The slides were subsequently incubated overnight with antibodies against CD68 (\#76437s, rabbit anti-human monoclonal, 1:400; Cell Signaling Technology) and FCER1G (ab151986, rabbit anti-human polyclonal, 1:400; Abcam) at $4^{\circ} \mathrm{C}$ in an incubator. After incubation with horseradish peroxidase-conjugated secondary antibodies (ab205718, goat anti-rabbit IgG polyclonal, 1:2000; Abcam) for 1 hour at room temperature the following day, slides were developed using 3,3'-diaminobenzidine staining for 2 min, and the cell nucleus was stained with hematoxylin. Human tonsil and spleen tissues were used as positive controls for CD68 and FCER1G, respectively (Data not shown). The ccRCC slides incubated only with secondary antibodies were used as negative control (Data not shown). All slides were scanned with Hamamatsu Nanozoomer S60 (Hamamatsu City, Japan), and evaluated on the same displayer using the NDP.view software (Version 2.9.20). Given that both CD68 and FCER1G are membrane surface markers that label single cells within tumors, the staining intensity was defined as the percentage of area covered by positive cells $(0-100)$ multiplied by the density of positive cells in given areas $(0-3)$. Using this standard, an IHC score of $0-300$ was generated for statistical evaluation. All slides were independently evaluated by two experienced pathologists, who calculated the mean IHC score. The representative images were captured using Olympus microscope (model BX51) and processed using Adobe Photoshop and Illustrator (Version CC 2017).

\section{Statistical analysis}

The two-tailed Student's $t$-test or Wilcoxon test was conducted for continuous variables. The chi-squared test or Fisher's exact test was conducted for categorical variables. The Kaplan-Meier method was used to draw survival curves using the "survival" package and "survminer" in $\mathrm{R}$ software version 3.6.3. Variables with a P-value $<0.05$ on the univariate analysis were included in the multivariate Cox regression analysis using the "survival" package of $\mathrm{R}$ 3.6.3. Receiver operating characteristics (ROC) analysis was performed to obtain the cut-off value and the area under the ROC curve (AUC) using the GraphPad Prism 7.00 software (GraphPad Software, Inc., San Diego, CA, USA). The prognostic accuracy of the strata and other clinical prognostic factors was calculated through Harrell's concordance index analysis using the "survcomp" package in R software version 3.6.3. Nomogram analysis was conducted using the "foreign" (version 0.8-78) and "rms" (version 6.0.1) packages to establish the risk prediction model. All statistical analyses were performed using the R software (version 3.6.3) and GraphPad Prism (version 7.00).

\section{Results}

Identification of macrophage-related DEGs in ccRCC using TCGA-KIRC database

TCGA-KIRC data, including 538 tumors and 72 NATs, were downloaded using the TCGAbiolinks R-package [18]. For data preprocessing, 167 tumor samples were excluded based on the standard of $<60 \%$ tumor purity using the TCGAtumor_purity function of the package. The remaining data were further normalized using the GC-content method [19] and genes with expression levels $<25 \%$ of the average levels were excluded. After data preprocessing, the expression data of 13,125 genes from 363 tumor samples and 72 NATs were used for DEGs analysis using the R-package Limma. Using a threshold of the absolute value of log fold change $>0.1$ and an adjusted P-value $<1 \mathrm{e}-10$, it was discovered that 3,323 genes were differentially expressed between tumors and NATs (Fig. 1). Moreover, we selected the five most cited [22-25] macrophage biomarkers (i.e., CD14, CD68, $C D 86, C D 163$, and colony stimulating factor 1 receptor [CSF1R]) and calculated their Pearson's correlation coefficient with the discovered DEGs. Thus, a set of correlated DEGs was generated for each of the five macrophage biomarkers. For the detection of genes closely related to TAMs, correlated DEGs with a Pearson's correlation coefficient $>0.4$ and $P$-value $<1 \mathrm{e}-10$ were considered strongly correlated DEGs (An additional table file shows this in more detail (see Additional file 3)). The Venn diagram intersection analysis revealed that five candidate DEGs were closely related to all selected macrophage biomarkers, namely SH3 and cysteine rich domain 3 (STAC3), galectin 9 (LGALS9), triggering receptor expressed on myeloid cells 2 (TREM2), Fc fragment of IgE receptor Ig (FCER1G), and paired immunoglobin like type 2 receptor alpha (PILRA) (Fig. 2A, B). For validation of our findings, the web-based tool TIMER 2.0 [26] was employed to calculate the correlation score between the abundance of macrophage infiltrates in ccRCC and the expression levels of each candidate DEG using TCGA-KIRC dataset. 


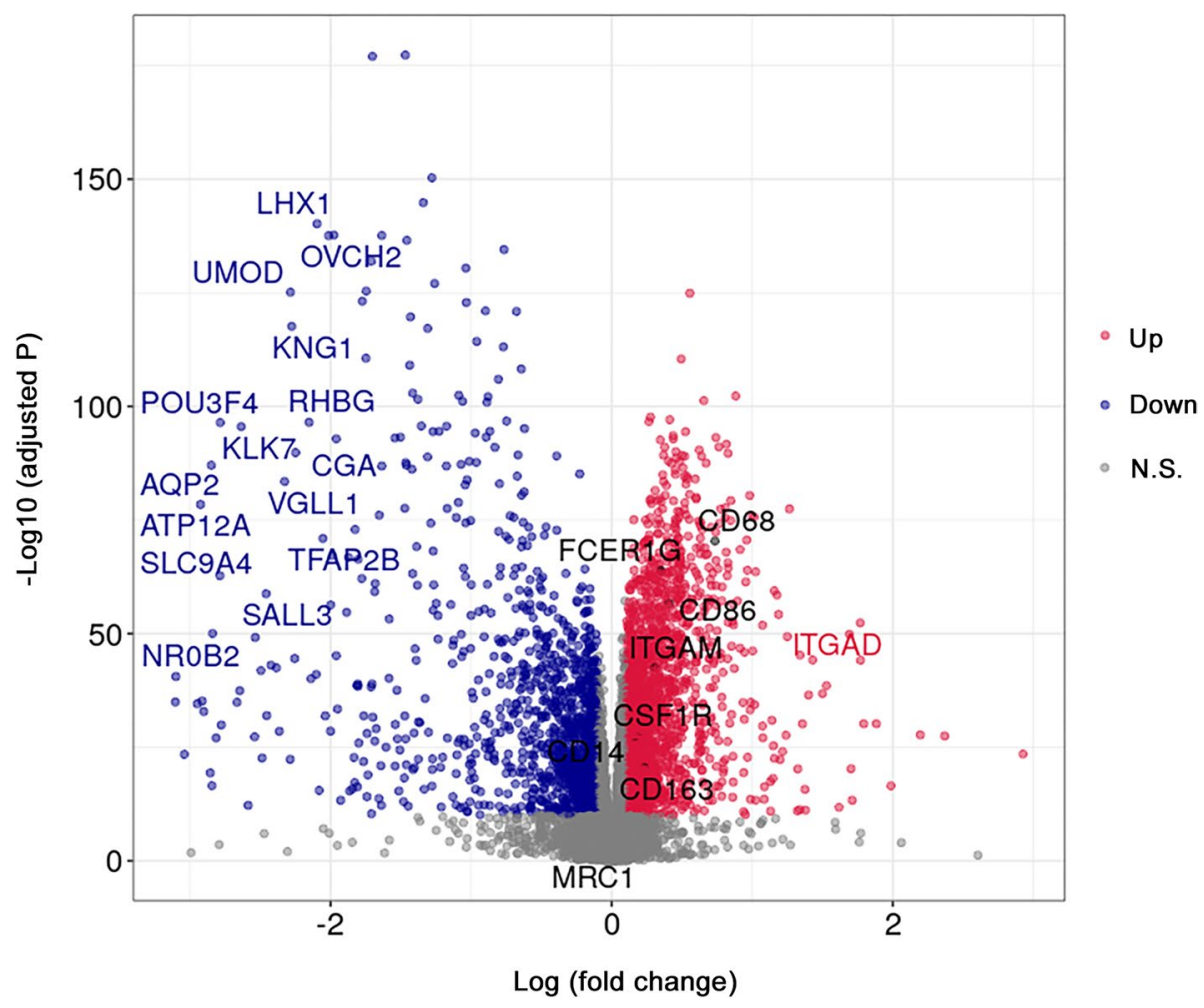

Fig. 1 The differentially expressed genes (DEGs) in TCGA-KIRC database. Volcano plot of DEGs between tumor and NATs identified in ccRCC. The red and blue points in the plot represent DEGs with statistical significance $(|\log F C|>0.1$, adjusted $P<1$ e-10) N.S.: No significance. ccRCC, clear cell renal cell carcinoma; logFC, log fold change; NATs, normal tissues adjacent to tumor; TCGA-KIRC, The Cancer Genome Atlas-kidney renal clear cell carcinoma;

For each entered candidate DEG, $\geq 13$ of 15 algorithms showed a positive correlation $(P<0.05, \rho>0)$ (Fig. 2 C).

\section{FCER1G was abundantly expressed in CcRCC} and contributed to poor prognosis in patients receiving anti-PD-1 treatment

To determine their clinical importance, patients with ccRCC from TCGA-KIRC database were divided into two groups according to the median expression levels of each candidate DEG. A Kaplan-Meier survival curve was drawn to examine the difference in OS between the two groups. Among the five identified macrophagerelated DEGs, only STAC3 $(P=0.00073)$ and FCER1G $(P=0.0068)$ showed a significant $(P<0.05)$ prognostic difference between the groups (Fig. 3A). Of note, patients with different STAC3 expression levels exhibited a more significant diversity in OS. However, STAC3 showed the lowest average expression in ccRCC tissue among all five DEGs (Fig. 3B). Therefore, we selected FCER1G as the most pronounced macrophage-related DEG with prognostic value. To further investigate the prognostic value of FCER1G, we downloaded the bulk RNA-seq and clinical data of the CheckMate 025 clinical trial, based on which Nivolumab (anti-PD-1) was approved by the Food and Drug Administration for the treatment of ccRCC [20]. In this dataset, 181 and 130 patients with ccRCC were treated with Nivolumab and Everolimus (MTOR inhibitor), respectively. The best cut-off value of FCER1G for each group was derived by the ROC curve (Data not shown). The ROC curve revealed that the best cut-off value for FCER1G expression in Nivolumab arm was 33.34. For Everolimus arm, The ROC curve showed that the best cut-off value for FCER1G expression was 34.27. The Kaplan-Meier survival curve showed that following the administration of Nivolumab, patients with a higher FCER1G expression levels were associated with a worse prognosis (Fig. 3C). In contrast, this tendency was not evident in those treated with Everolimus (Fig. 3D).

\section{FCER $1 G$ and macrophage markers exerted a synergistic effect on predicting the survival of patients with cCRCC, and high FCER1G expression was related to suppression of $T$ lymphocytes}

To the best of our knowledge, there are no studies exploring the exact role of FCER1G in ccRCC tumor immunity. According to the above-mentioned results, we 

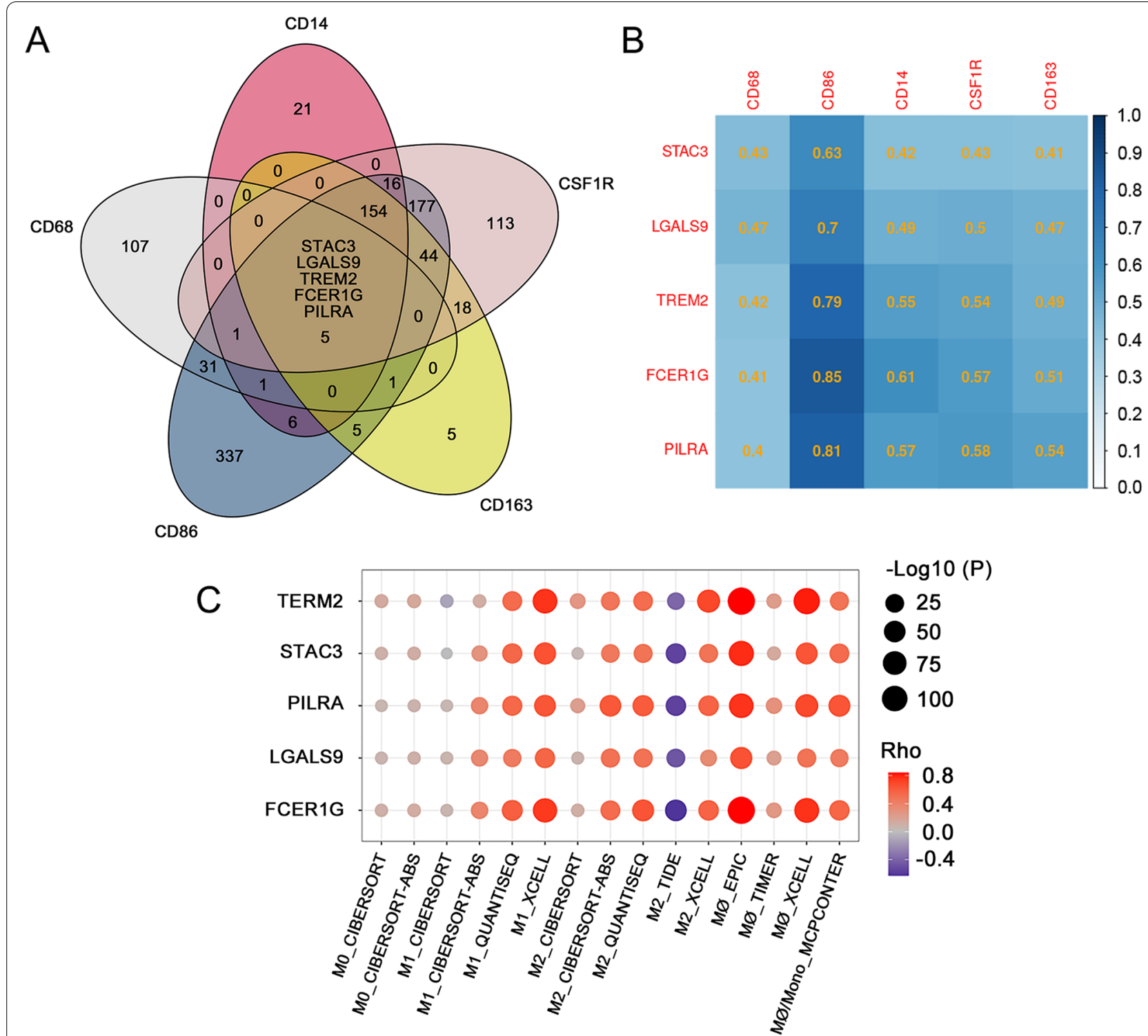

Fig. 2 The correlation between DEGs and macrophage markers in TCGA-KIRC database. A The Venn diagram shows the intersection of DEGs related to macrophage markers; each ellipse represents a DEG set strongly correlated with a given macrophage marker on the vertex $(r>0.4, P<1$ e-10). B The heat map shows the Pearson's correlation coefficients of five candidate DEGs with macrophage markers. C The bubble chart shows the strong correlation of five candidate DEGs with macrophage infiltration in cCRCC estimated using TIMER 2.0. ccRCC, clear cell renal cell carcinoma; DEGs, differentially expressed genes; TCGA-KIRC, The Cancer Genome Atlas-kidney renal clear cell carcinoma

(See figure on next page.)

Fig. 3 FCER1G was abundantly expressed in CCRCC and indicated poor prognosis in patients receiving anti-PD-1 treatment. A Kaplan-Meier curves revealing that high expression of STAC3 and FCER1G were related to poor overall survival in patients with cCRCC from TCGA-KIRC. B The violin plot shows that STAC3 has the lowest expression levels among the five candidate DEGs. CThe Kaplan-Meier survival curve shows that high FCER1G expression was related to poor overall survival in patients treated with Nivolumab in the CheckMate 025 clinical trial, but not in $\mathbf{D}$ those treated with Everolimus in the same research study. ccRCC, clear cell renal cell carcinoma; DEG, differentially expressed gene; FCER1G, Fc fragment of IgE receptor Ig; PD-1, programmed cell death 1; STAC3, SH3 and cysteine rich domain 3; TCGA-KIRC, The Cancer Genome Atlas-kidney renal clear cell carcinoma 

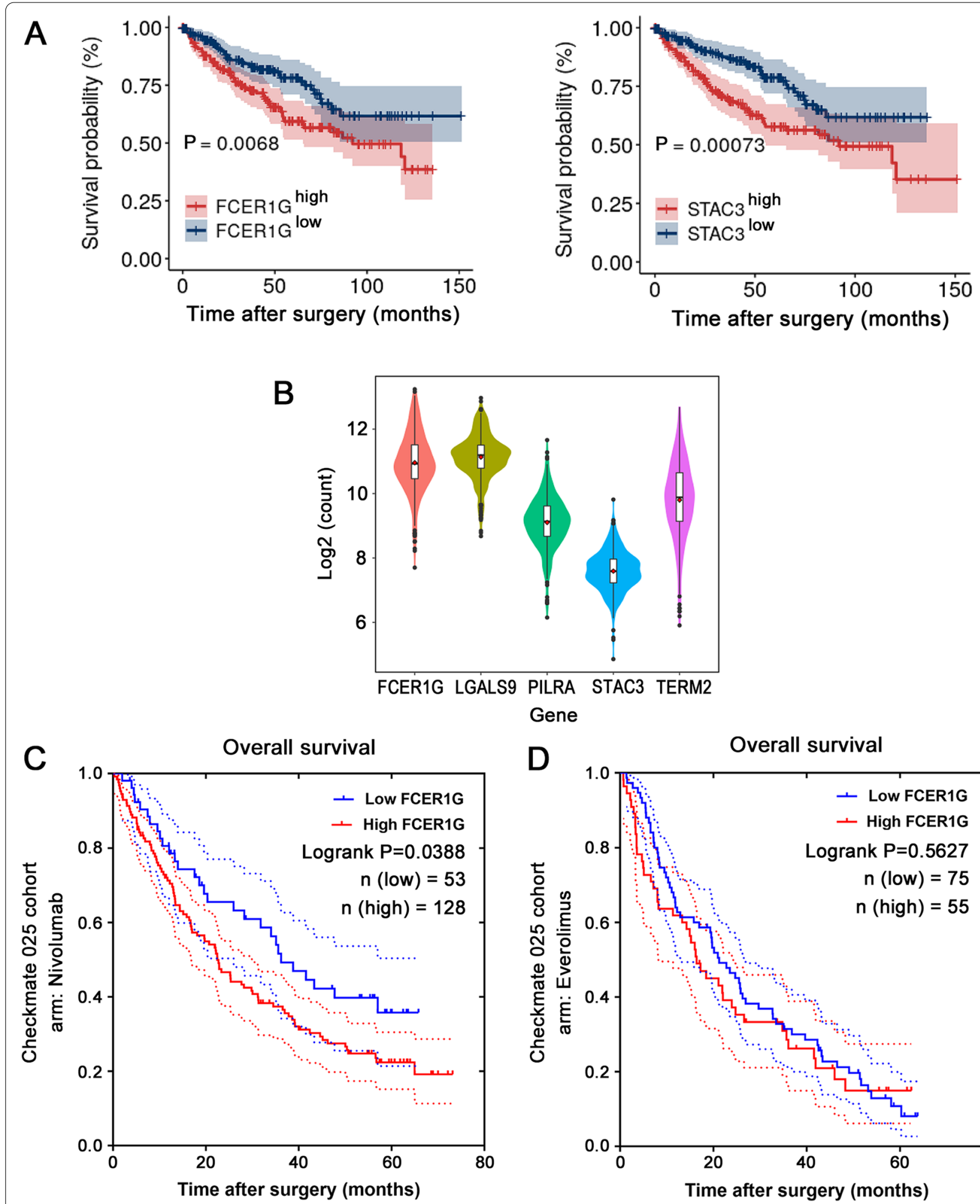

Fig. 3 (See legend on previous page.)

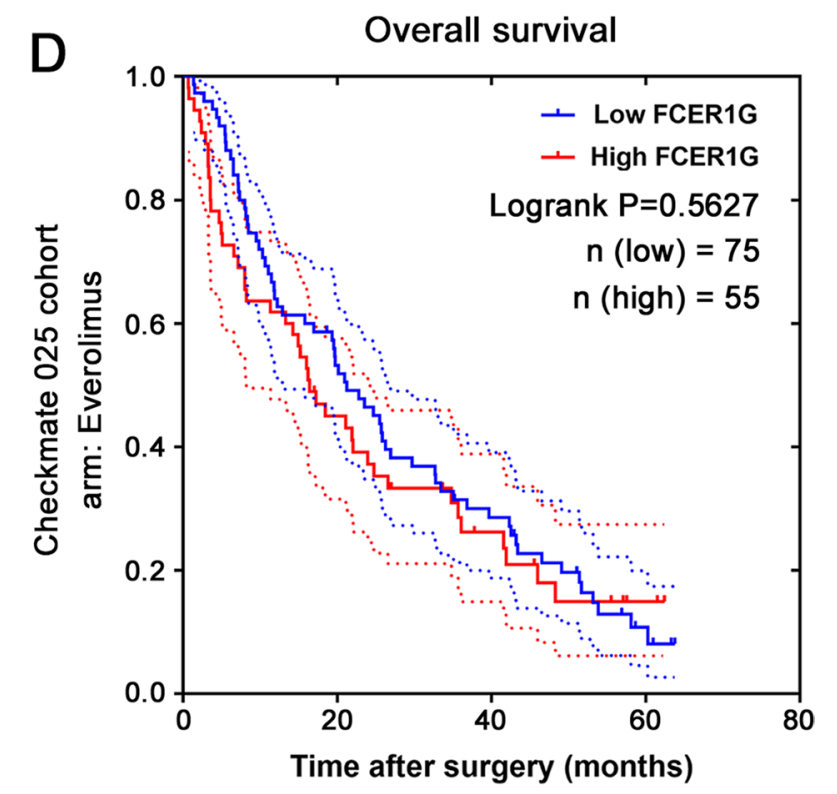


investigated the potential synergistic effect of FCER1G expression and macrophage presence in ccRCC. We employed the median expression levels of FCER1G and macrophage markers to classify patients from TCGAKIRC database into four expression groups: FCER1G ${ }^{\text {high }}$, Marker high; FCER1G ${ }^{\text {high }}$, Marker ${ }^{\text {low }}$ FCER1G ${ }^{\text {low }}$, Mark$e r^{\text {high }}$ and FCER $1 G^{\text {low }}$, Marker ${ }^{\text {low }}$. In the groups using CD68 (Fig. 4A) and CD163 (Fig. 4B) as classification markers, the combined indicator showed better survival stratification in the Kaplan-Meier survival method. The best stratification was observed with the combination of CD68 and FCER1G (Fig. 4B). Patients with low expression of both FCER1G and CD68 had the longest OS, whereas those with high expression of both FCER1G and $C D 68$ were linked to the worst clinical prognosis. To further investigate the underlying mechanisms of FCER $1 G$ in ccRCC, we performed GSEA by mapping the gene phenotype plotted according to the FCER $1 G$ expression
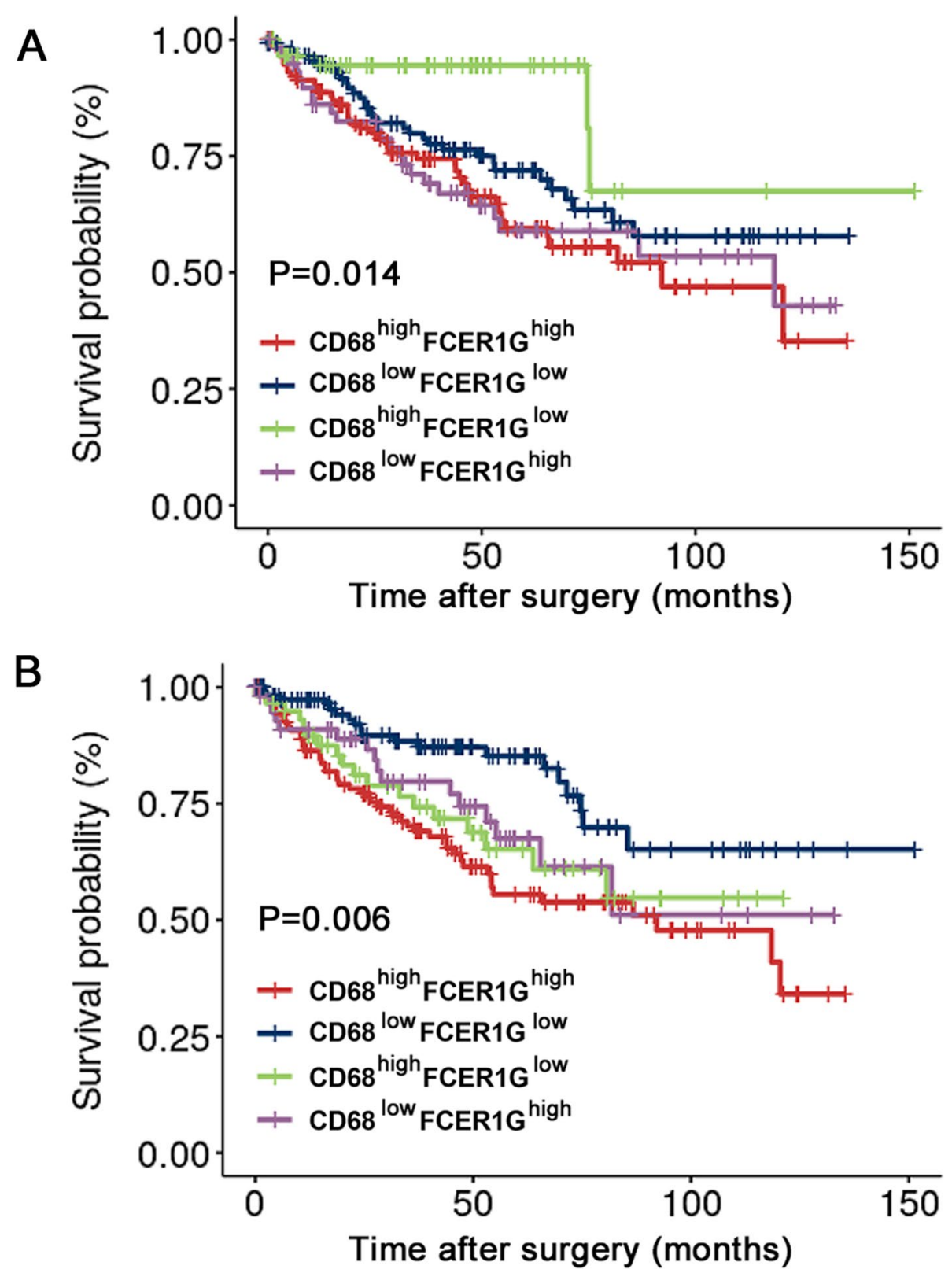

Fig. 4 FCER1G and macrophage markers exerted a synergistic effect in predicting ccRCC survival. The Kaplan-Meier survival curve shows the combination of FCER1G and macrophage marker CD163 (A) or CD68 (B) better predicts patient survival in TCGA-KIRC. ccRCC, clear cell renal cell carcinoma; FCER1G, Fc fragment of IgE receptor Ig;TCGA-KIRC, The Cancer Genome Atlas-kidney renal clear cell carcinoma 


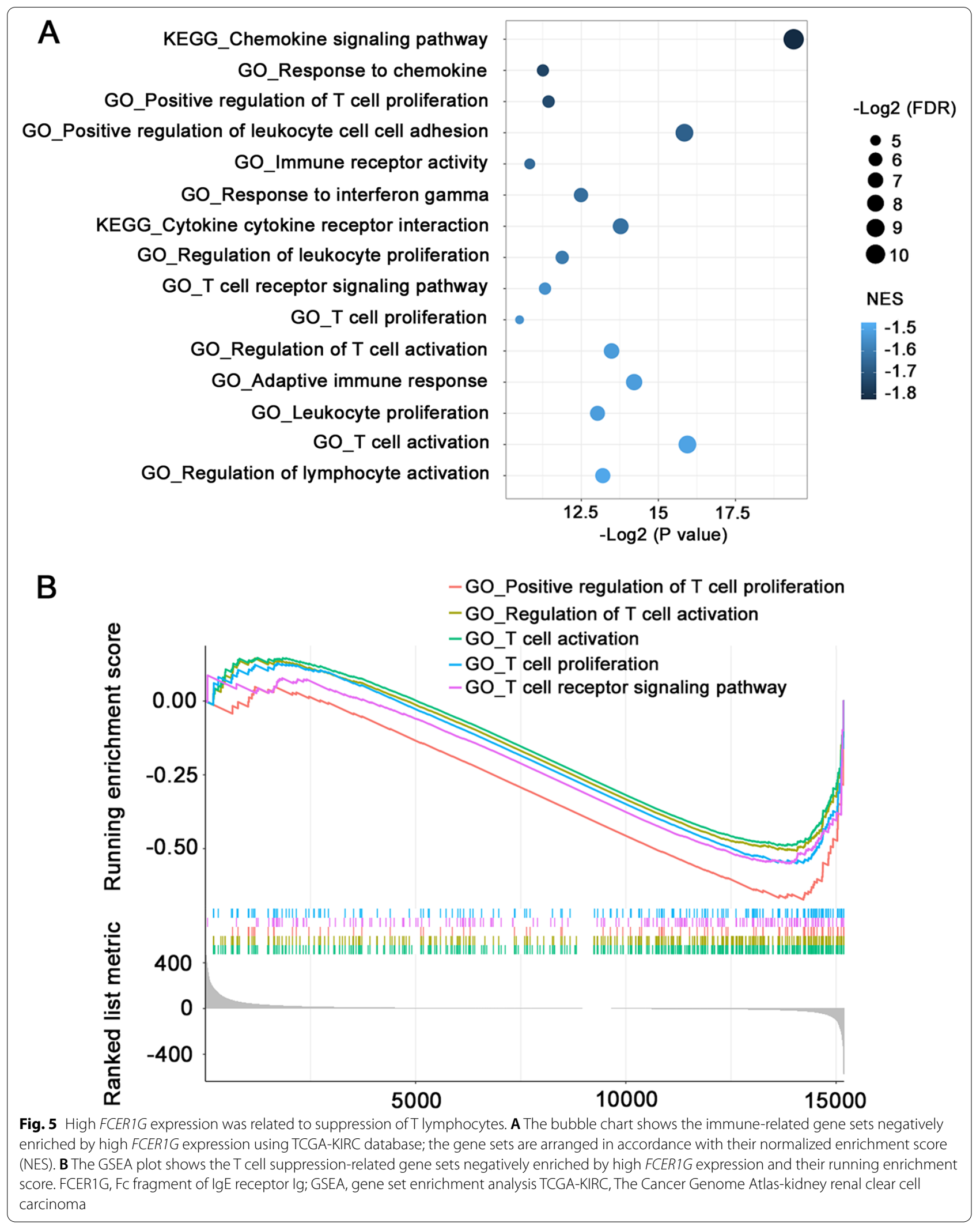




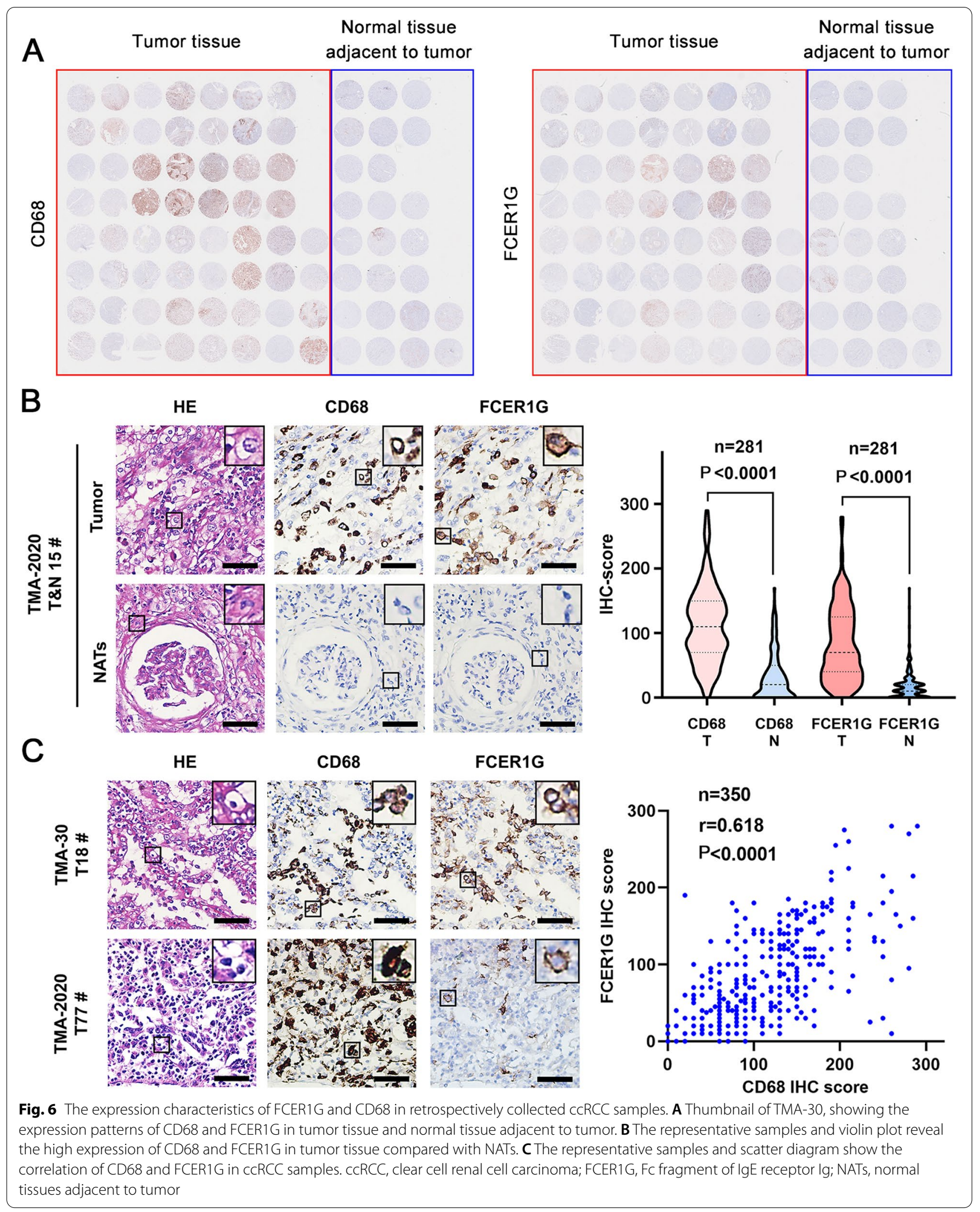


levels in TCGA-KIRC with the data obtained from the Gene Ontology and Kyoto Encyclopedia of Genes and Genomes database. Only gene sets with false discovery rate and nominal P-values $<0.05$ were considered significantly enriched (An additional table file shows this in more detail (see Additional file 5)). Most gene sets negatively enriched by high FCER1G expression were related to immune response (Fig. 5A). Notably, gene sets related to $\mathrm{T}$ cell function were the most common among all immune response gene sets (Fig. 5B). The results of the GSEA suggested that high FCER1G expression in ccRCC is functionally correlated with the suppression of $\mathrm{T}$ lymphocytes. This finding is also consistent with the survival curve of patients treated with Nivolumab in the CheckMate 025 trial.

\section{Prognostic value of FCER1G and CD68 in retrospectively collected ccRCC samples}

To further validate our findings in clinical setting, we collected samples from 350 patients with ccRCC (Table 1) (Additional table files show this in more detail (see Additional file 1 and 2)) who underwent either open or laparoscopic surgery from 2010 to 2018 in Shanghai Changhai Hospital. Samples stained with hematoxylineosin were examined, and nine pieces of TMA were produced (TMA-30 and TMA-2020 NO.1-8, described in Methods). IHC staining was performed to determine the expression of FCER1G and CD68 in ccRCC using successive sections (thickness: $4 \mu \mathrm{m}$ ) (Fig. 6A). Next, the IHC-score (described in Methods) of each sample was evaluated. Higher expression of both FCER1G and CD68 was detected in tumors compared with NATs (Fig. 6B). Meanwhile, the expression levels of FCER1G and CD68 were strongly correlated in ccRCC tissues (Fig. 6C), with a Pearson's correlation coefficient of 0.618. Although the correlation of expression was strong, we observed that the expression pattern of FCER1G and CD68 differed in a considerable portion of samples (Fig. 6A, C lower left); hence, the types of cells that FCER1G and CD68 represent varied. Owing to the long duration of the follow-up and completeness of OS and PFS, TMA- 30 was used as the training group to determine the best cut-off value for the FCER1G and CD68 IHC-score. Using 5-year OS as primary outcome, the ROC curve showed that the best IHC-score cut-off value for FCER1G was 77.5, with an AUC of 0.7026. For CD68, the best IHC-score cut-off value was 147.5, with an AUC of 0.8237 (Fig. 7) (Tables 2, 3). Subsequently, a Kaplan-Meier survival curve was drawn for TMA-2020 NO.1-8 to evaluate the prognostic value of FCER1G or CD68. Patients with high expression levels of FCER1G (Fig. 8A) or CD68 (Fig. 8B) were associated with inferior OS and PFS. Univariate and multivariate Cox regression analyses were performed to further determine whether FCER1G and CD68 were independent risk factors for evaluating OS and PFS in patients with ccRCC. Both FCER1G and CD68 were associated

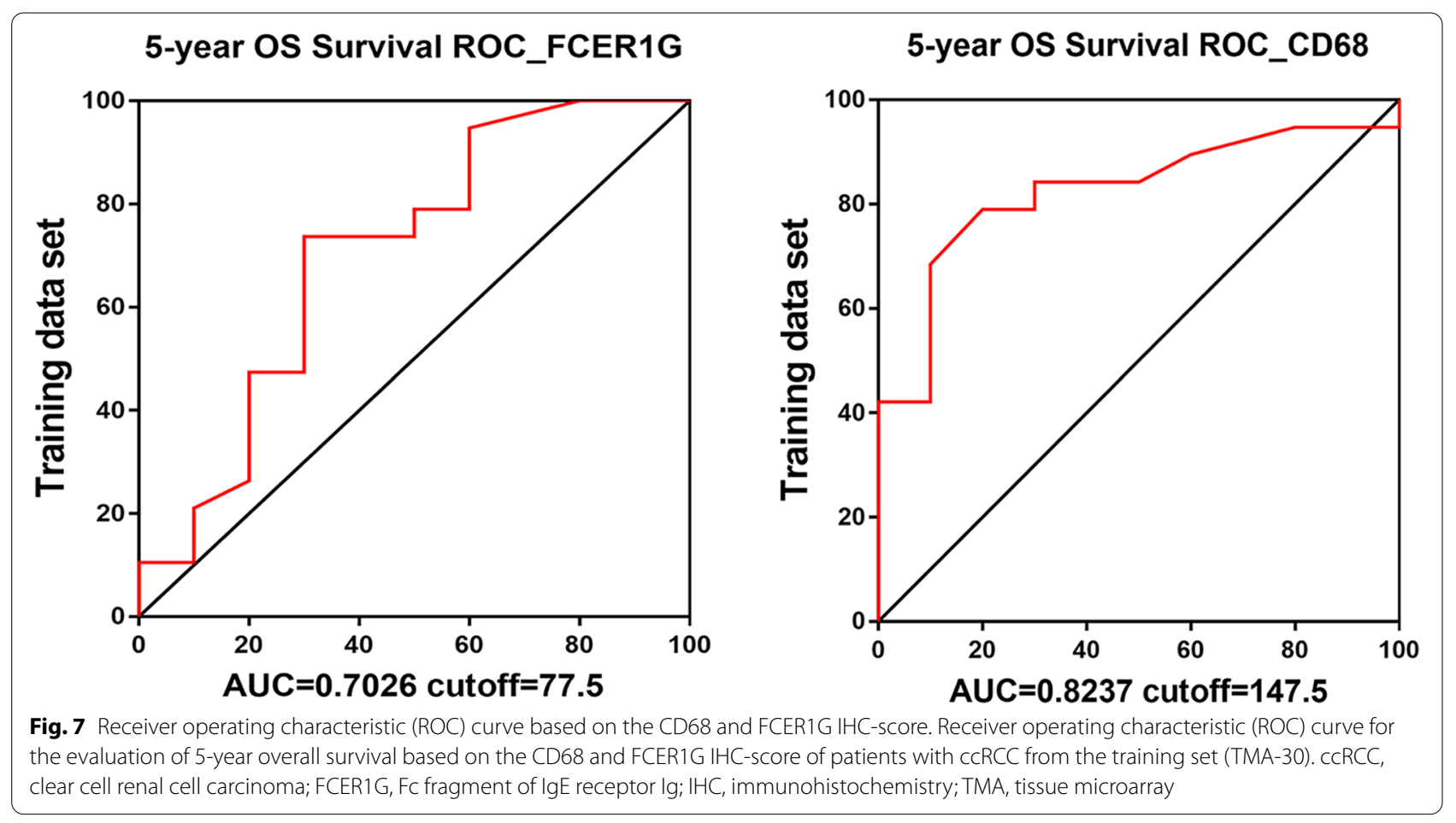


Table 2 Correlation between FCER1G expression and clinical characteristics of patients with CCRCC in TMA-2020 NO.1-8 $(n=321)$

\begin{tabular}{|c|c|c|c|c|}
\hline \multirow[t]{2}{*}{ Characteristics } & \multicolumn{2}{|c|}{$\begin{array}{l}\text { FCER1G expression in } \\
\text { TMA-2020 NO.1-8 }\end{array}$} & \multirow[t]{2}{*}{$\begin{array}{l}\text { Total } \\
(n=321)\end{array}$} & \multirow[t]{2}{*}{$P$-value } \\
\hline & $\begin{array}{l}\text { High } \\
\text { expression } \\
(n=177)\end{array}$ & $\begin{array}{l}\text { Low } \\
\text { expression } \\
(n=144)\end{array}$ & & \\
\hline Age & & & & 0.8798 \\
\hline$<60$ years & 103 & 85 & 188 & \\
\hline$\geq 60$ years & 74 & 59 & 133 & \\
\hline Sex & & & & 0.0663 \\
\hline Male & 116 & 108 & 224 & \\
\hline Female & 61 & 36 & 97 & \\
\hline Fuhrman grade & & & & $<0.0001$ \\
\hline $1-2$ & 158 & 103 & 261 & \\
\hline $3-4$ & 19 & 41 & 60 & \\
\hline TNM stage & & & & 0.0046 \\
\hline$|-| \mid$ & 161 & 114 & 275 & \\
\hline III-IV & 15 & 30 & 45 & \\
\hline NA & 1 & 0 & 1 & \\
\hline T category & & & & 0.0008 \\
\hline T1a-T1b & 152 & 100 & 252 & \\
\hline $\mathrm{T} 2 \mathrm{a}-\mathrm{T} 4$ & 24 & 44 & 68 & \\
\hline NA & 1 & 0 & 1 & \\
\hline Overall survival & & & & 0.0291 \\
\hline- & 141 & 108 & 249 & \\
\hline+ & 5 & 12 & 17 & \\
\hline NA & 31 & 24 & 55 & \\
\hline $\begin{array}{l}\text { Progression-free } \\
\text { survival }\end{array}$ & & & & 0.0010 \\
\hline- & 138 & 98 & 236 & \\
\hline+ & 8 & 22 & 30 & \\
\hline NA & 31 & 24 & 55 & \\
\hline
\end{tabular}

cCRCC clear cell renal cell carcinoma; FCER1G Fc fragment of lgE receptor Ig; NA not applicable; TMA tissue microarray

with shorter OS and PFS in Univariate Cox regression analysis. Following multivariable adjustment (i.e., age, sex, Fuhrman grade, TNM stage, and T category), CD68 was identified as an independent risk factor for the PFS of patients with ccRCC (Tables 4,5$)$.

\section{Combination of CD68 and FCER1G expression resulted} in a better prognostic stratification in patients with CCRCC To further test the possible synergistic effect of FCER1G and CD68 expression in predicting the prognosis of ccRCC, 321 patients in TMA-2020 were classified into four groups using the cut-off values determined from the ROC curve: FCER1G ${ }^{\text {high }}, \mathrm{CD}^{\text {high }}$; FCER1G ${ }^{\text {high }}$,

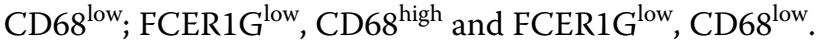
The Kaplan-Meier survival curve showed a significant
Table 3 Correlation between CD68 expression and clinical characteristics of patients with cCRCC in TMA-2020 NO.1-8 $(n=321)$

\begin{tabular}{|c|c|c|c|c|}
\hline \multirow[t]{2}{*}{ Characteristics } & \multicolumn{2}{|c|}{$\begin{array}{l}\text { CD68 expression in } \\
\text { TMA-2020 NO.1-8 }\end{array}$} & \multirow[t]{2}{*}{$\begin{array}{l}\text { Total } \\
(\mathrm{n}=321)\end{array}$} & \multirow[t]{2}{*}{$P$-value } \\
\hline & $\begin{array}{l}\text { High } \\
\text { expression } \\
(n=238)\end{array}$ & $\begin{array}{l}\text { Low } \\
\text { expression } \\
(n=83)\end{array}$ & & \\
\hline \multicolumn{3}{|l|}{ Age } & & 0.4993 \\
\hline$<60$ years & 142 & 46 & 188 & \\
\hline$\geq 60$ years & 96 & 37 & 133 & \\
\hline \multicolumn{4}{|l|}{ Sex } & 0.0914 \\
\hline Male & 160 & 64 & 224 & \\
\hline Female & 78 & 19 & 97 & \\
\hline \multicolumn{4}{|l|}{ Fuhrman grade } & $<0.0001$ \\
\hline $1-2$ & 207 & 54 & 261 & \\
\hline $3-4$ & 31 & 29 & 60 & \\
\hline \multicolumn{4}{|l|}{ TNM stage } & 0.0024 \\
\hline$|-| \mid$ & 213 & 62 & 275 & \\
\hline III-IV & 24 & 21 & 45 & \\
\hline NA & 1 & 0 & 1 & \\
\hline \multicolumn{4}{|l|}{ T category } & 0.0117 \\
\hline T1a-T1b & 196 & 56 & 252 & \\
\hline $\mathrm{T} 2 \mathrm{a}-\mathrm{T} 4$ & 41 & 27 & 68 & \\
\hline NA & 1 & 0 & 1 & \\
\hline \multicolumn{4}{|l|}{ Overall survival } & 0.0282 \\
\hline- & 191 & 58 & 249 & \\
\hline+ & 9 & 8 & 17 & \\
\hline NA & 38 & 17 & 55 & \\
\hline \multicolumn{4}{|c|}{ Progression-free survival } & $<0.0001$ \\
\hline- & 186 & 49 & & \\
\hline+ & 14 & 16 & & \\
\hline NA & 38 & 17 & 55 & \\
\hline
\end{tabular}

cCRCC clear cell renal cell carcinoma; NA not applicable; TMA tissue microarray

difference in survival between different groups in terms of OS (Fig. 9A) and PFS (Fig. 9B). The group with high expression of both FCER1G and CD68 showed the worst prognosis (Fig. 9C). Furthermore, we examined the prognostic accuracy of FCER1G and CD68 expression versus that of established indicators in patients with ccRCC. Concordance index analysis was used in the validation cohort $(n=321)$, which demonstrated that the integration of CD68 and FCER1G expression into the established prognostic indicators exhibited a higher concordance index value than any of these indicators alone (Table 6). In the same TNM stage group, patients with high expression of both CD68 and FCER1G (double high) showed worse OS (Fig. 10A) and PFS (Fig. 10B) compared with the remaining patients (non-double 

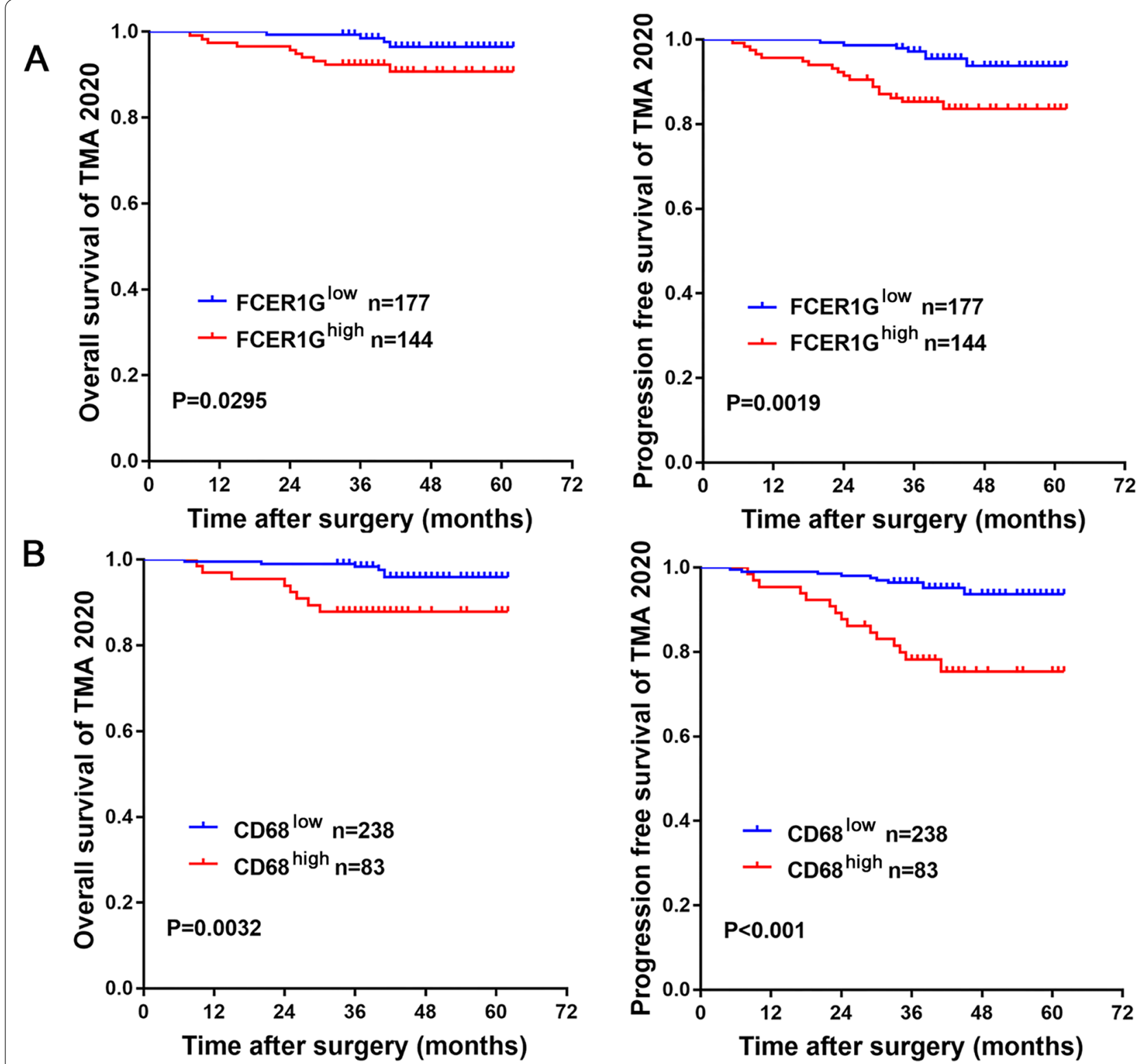

Fig. 8 Prognostic value of FCER1G and CD68 in retrospectively collected ccRCC samples. A The Kaplan-Meier survival curve shows that high expression of FCER1G was related to poor overall survival and progression-free survival in patients from the validation set (TMA-2020). B The Kaplan-Meier survival curve shows that high expression of CD68 was related to poor overall survival and progression-free survival in patients from the validation set (TMA-2020). ccRCC, clear cell renal cell carcinoma; FCER1G, Fc fragment of IgE receptor Ig; IHC, immunohistochemistry; TMA, tissue microarray

high). This may assist physicians in distinguish high-risk patients following surgery for ccRCC. Finally, we constructed nomograms to predict OS and PFS in patients with ccRCC at 3 and 5 years (Fig. 11A). Calibration plots of the nomograms for the prediction of 3- and 5-year OS (Fig. 11B) and PFS (Fig. 11C) are presented below.

Discussion In this study, we reported five DEGs in ccRCC that are strongly correlated with the expression of macrophage markers (i.e., LGALS9, PILRA, TREM2, STAC3, and FCER1G). LGALS9 encodes galectin-9 which is a beta-galactoside-binding protein participating in cell-cell and cell-matrix interactions [27]. PILRA encodes PILR $\alpha$, which is an immune inhibitory receptor possessing an immunoreceptor tyrosine-based inhibitory motif (ITIM) in its cytoplasmic domain [28]. TERM2, the coding gene for triggering a receptor expressed on 
Table 4 Univariate and multivariate Cox regression analyses of patient characteristics with overall survival

\begin{tabular}{|c|c|c|c|c|}
\hline \multirow[t]{2}{*}{ Characteristics } & \multicolumn{2}{|l|}{ Univariate } & \multicolumn{2}{|l|}{ Multivariate } \\
\hline & $\mathrm{HR}(95 \% \mathrm{Cl})$ & $P$-value & $\mathrm{HR}(95 \% \mathrm{Cl})$ & $P$-value \\
\hline Age ( $<60$ vs. $\geq 60$ years) & $2.1(0.72-6.00)$ & 0.18 & & \\
\hline Sex (female vs. male) & $1.6(0.44-5.70)$ & 0.48 & & \\
\hline Fuhrman grade (1-2 vs. $3-4)$ & $4.5(1.6-13.0)$ & 0.005 & $4.027(1.3926-11.6430)$ & 0.0101 \\
\hline TNM stage (1-2 vs. $3-4)$ & $4.8(1.6-14.0)$ & 0.0049 & $3.092(0.9729-9.8270)$ & 0.0557 \\
\hline T category (T1a-1b vs. T2a-T4) & $6.1(2.9-13.0)$ & $1.5 \mathrm{E}-06$ & $5.0043(1.8022-13.8960)$ & 0.002 \\
\hline CD68 (low vs. high) & $4.3(1.5-12.0)$ & 0.0069 & $2.5303(0.7912-8.0920)$ & 0.11758 \\
\hline FCER1G (low vs. high) & $3.4(1.1-11.0)$ & 0.04 & $1.5414(0.4111-5.7790)$ & 0.52102 \\
\hline
\end{tabular}

Cl confidence interval; FCER1G Fc fragment of IgE receptor Ig; $H R$ hazard ratio

Table 5 Univariate and multivariate Cox regression analyses of patient characteristics with progression-free survival

\begin{tabular}{|c|c|c|c|c|}
\hline \multirow[t]{2}{*}{ Characteristics } & \multicolumn{2}{|l|}{ Univariate } & \multicolumn{2}{|l|}{ Multivariate } \\
\hline & $\mathrm{HR}(95 \% \mathrm{Cl})$ & $P$-value & $\mathrm{HR}(95 \% \mathrm{Cl})$ & $P$-value \\
\hline Age (<60 vs. $\geq 60$ years) & $1.5(0.69-3.10)$ & 0.32 & & \\
\hline Sex (female vs. male) & $1.5(0.6-3.7)$ & 0.38 & & \\
\hline Fuhrman grade (1-2 vs. $3-4)$ & $3.9(1.8-8.2)$ & 0.0005 & $3.621(1.6718-7.8450)$ & 0.0011 \\
\hline TNM stage (1-2 vs. $3-4)$ & $6.6(3-14)$ & 1.7E-06 & $4.886(2.1503-11.1010)$ & 0.00015 \\
\hline T category (T1a-1b vs. T2a-T4) & $5.5(3.3-9.1)$ & $1 \mathrm{E}-10$ & $3.7106(1.6951-8.1230)$ & 0.00104 \\
\hline CD68 (low vs. high) & $4.9(2.3-11.0)$ & 0.000047 & $2.9592(1.2744-6.8710)$ & 0.0116 \\
\hline FCER1G (low vs. high) & $4(1.7-9.5)$ & 0.0016 & $1.7784(0.6478-4.8820)$ & 0.26382 \\
\hline
\end{tabular}

Cl confidence interval; FCER1G Fc fragment of IgE receptor Ig; $H R$, hazard ratio

monocytes 2 , is responsible for the uptake of $\beta$-amyloid oligomers by microglia [29]. These three genes are highly involved in immune-related pathways. However, it has been reported that STAC3 encodes an important molecule involved in excitation-contraction coupling that regulates the contraction of skeletal muscles [30]. Although STAC3 showed prognostic importance in TCGA-KIRC database, its underlying mechanism warrants further investigation.

FCER1G encodes the Fc receptor $\gamma$ chain, which was firstly reported in 1990 to be the third subunit of the high-affinity immunoglobulin $\mathrm{E}$ (IgE) receptor (Fce RI) [31]. Later, it was demonstrated that the Fc receptor $\gamma$ chain is a common component of Fc receptors widely expressed in different types of immune cells and participates in a variety of immune responses, such as phagocytosis and cytokine release [32]. Under physiological conditions, Fc receptors eliminate pathogens and antigens by binding to the crystallizable fragment of immunoglobulins. In contrast, under pathological conditions, they can lead to abnormal immune responses such as IgE-dependent allergy [33].
It has been reported that, in the viral infection process, FCER1G-expressing natural killer cells could respond to the over activation of $\mathrm{CD}^{+} \mathrm{T}$ cells and suppress their function [34]. A reduction in regulatory $\mathrm{T}$ cells was also observed in FCER1G -deficient mice [35]. Similarly, GSEA analysis revealed that high FCER1G expression is related to suppression of $\mathrm{T}$ cell activation and proliferation. In the CheckMate 025 trial, high FCER1G expression was suggestive of poor prognosis in patients treated with an anti-PD-1 agent which is a monoclonal antibody that acts by blocking inhibitory transmembrane protein expressed on $\mathrm{T}$ cells to in turn stimulate the anti-tumor immune response. However, this association was not observed in patients treated with an MTOR inhibitor that mainly targets the tumor itself. Thus, we suggest that FCER1G expression is related to $\mathrm{T}$ cell function in ccRCC. However, the specific regulatory mechanism of this effect needs to be further studied.

If the two genes are highly correlated, there is a possibility that the two genes are expressed in the same type of cell. The expression of the Fc receptor $\gamma$ chain in macrophages has been previously reported by others 


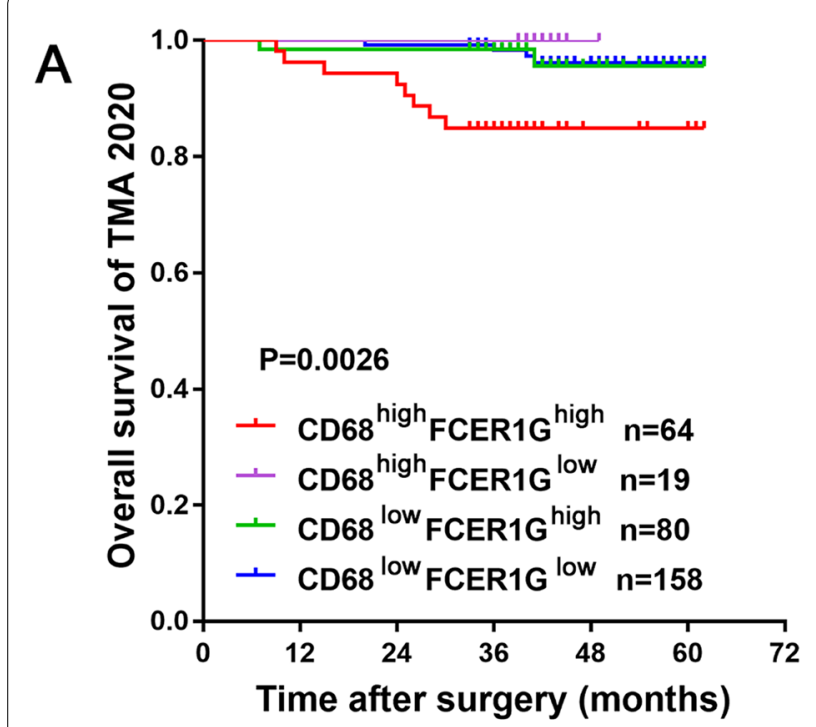

C

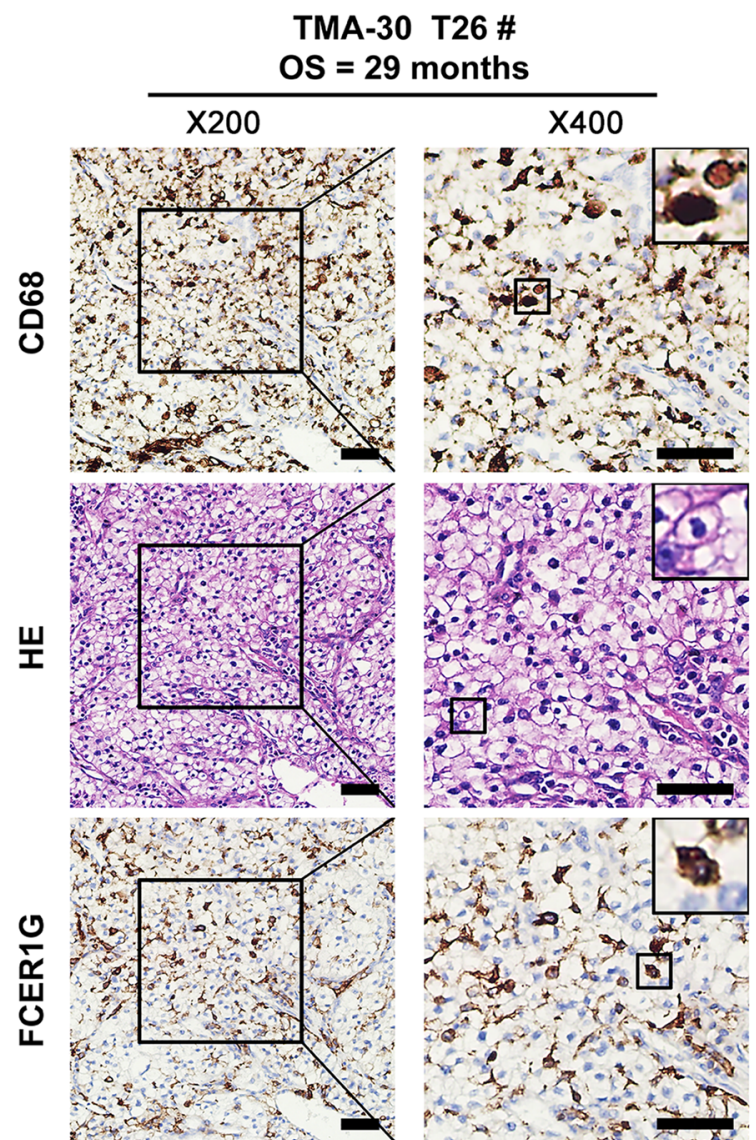

B
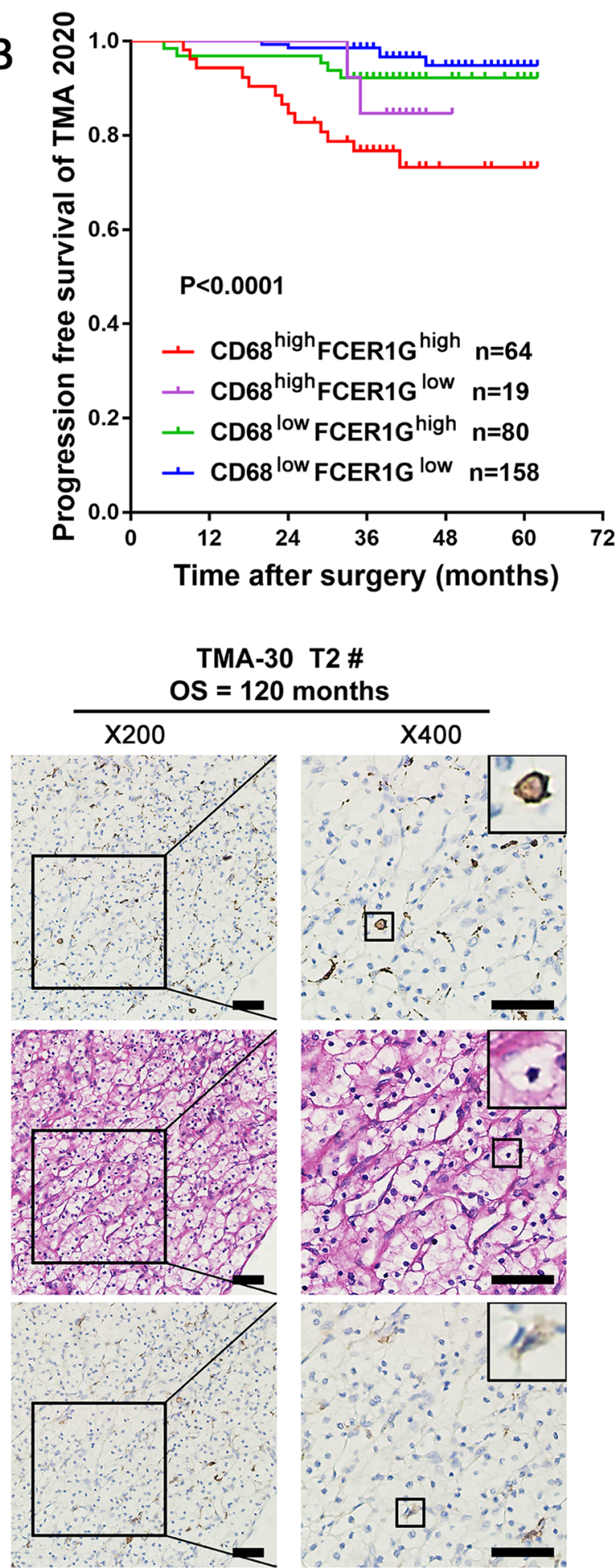

Fig. 9 Combining CD68 and FCER1G expression resulted in better prognostic stratification in patients with ccRCC. The Kaplan-Meier survival curve reveals that combining CD68 and FCER1G expression more accurately determines the overall survival (A) and progression-free survival (B) in patients with cCRCC from the validation set (TMA-2020). C Immunohistochemistry results of representative samples show an inferior overall survival in patients with high expression of both CD68 and FCER1G. cCRCC, clear cell renal cell carcinoma; FCER1G, Fc fragment of lgE receptor lg 
Table 6 Concordance index analysis

\begin{tabular}{lll}
\hline Characteristics & $\begin{array}{l}\text { Overall survival } \\
\text { Validation cohort }(\boldsymbol{n}=\mathbf{3 2 1})\end{array}$ & $\begin{array}{l}\text { Progression-free survival } \\
\text { Validation cohort }(\boldsymbol{n}=\mathbf{3 2 1})\end{array}$ \\
\hline Fuhrman grade (1-2 vs. 3-4) & $0.4970(0.4055-0.5884)$ & $0.4893(0.3917-0.5869)$ \\
TNM stage (1-2 vs. 3-4) & $0.5265(0.4153-0.6377)$ & $0.5217(0.3989-0.6444)$ \\
T category (T1a-1b vs T2a-T4) & $0.5419(0.4502-0.6335)$ & $0.5438(0.4439-0.6437)$ \\
CD68 (low vs. high) & $0.5616(0.4802-0.6431)$ & $0.5420(0.4546-0.6295)$ \\
CD68 + Fuhrman grade & $0.5236(0.4557-0.5917)$ & $0.5194(0.4480-0.5908)$ \\
CD68 + TNM stage & $0.5450(0.4743-0.6157)$ & $0.5404(0.4653-0.6156)$ \\
CD68 + T category & $0.5462(0.4795-0.6128)$ & $0.5451(0.4748-0.6155)$ \\
FCER1G (low vs. high) & $0.5959(0.5221-0.6696)$ & $0.5906(0.5141-0.6669)$ \\
FCER1G + Fuhrman grade & $0.5537(0.4922-0.6153)$ & $0.5653(0.4992-0.6314)$ \\
FCER1G + TNM stage & $0.5693(0.5050-0.6335)$ & $0.5676(0.5005-0.6348)$ \\
FCER1G + T category & $0.5686(0.5084-0.6288)$ & $0.5688(0.5059-0.6318)$ \\
CD68 + FCER1G & $0.5753(0.5145-0.6361)$ & $0.5668(0.5032-0.6305)$ \\
\hline
\end{tabular}

FCER1G, Fc fragment of IgE receptor Ig

[36, 37]. However, by IHC staining of the successive sections from our tissue microarray, we showed that the spatial orientation of FCER1G and CD68 differed in ccRCC, suggesting that FCER1G is located on macrophages and other types of cells. The Kaplan-Meier survival analysis also showed the worst outcome in the group with high mRNA and protein expression of both FCER1G and CD68, suggesting disparity in their function in FCER1G- and CD68-expressing cells. Therefore, it is important to examine the underlying molecular mechanism that governs the synergistic effect of FCER1G and CD68 in ccRCC. The Fc receptor $\gamma$ chain is an important component of allergy-related Fce RI, which is abundantly expressed on mast cells and basophils. It is established that the activation of Fce RI on mast cells and basophils leads to secretion of interleukin 4 (IL4) [38], which is an inducer of a tumor-suppressive macrophage subtype (M2 macrophage) [39]. In this rationale, the high expression of FCER $1 G$ in ccRCC could be a functional basis for the induction of M2

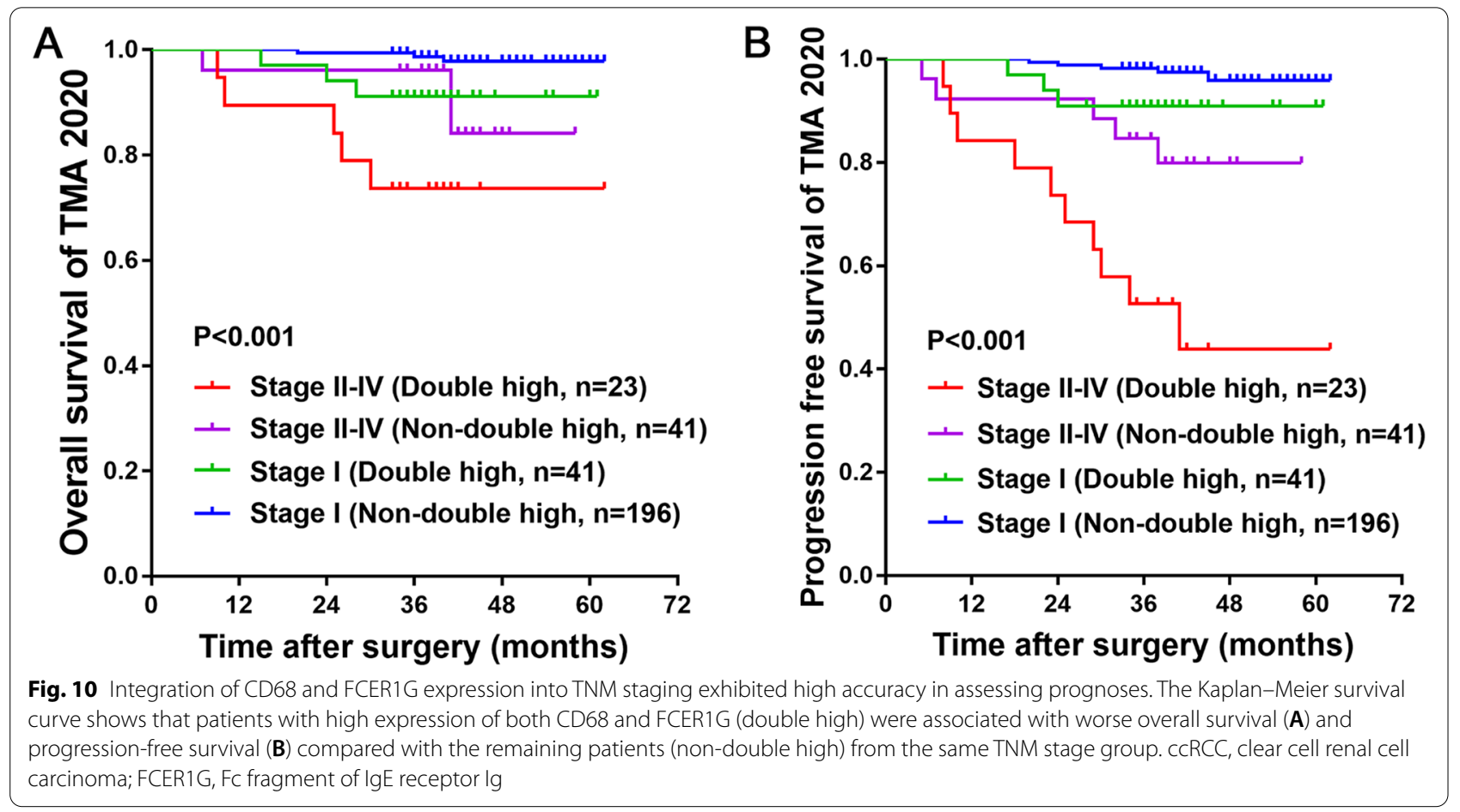




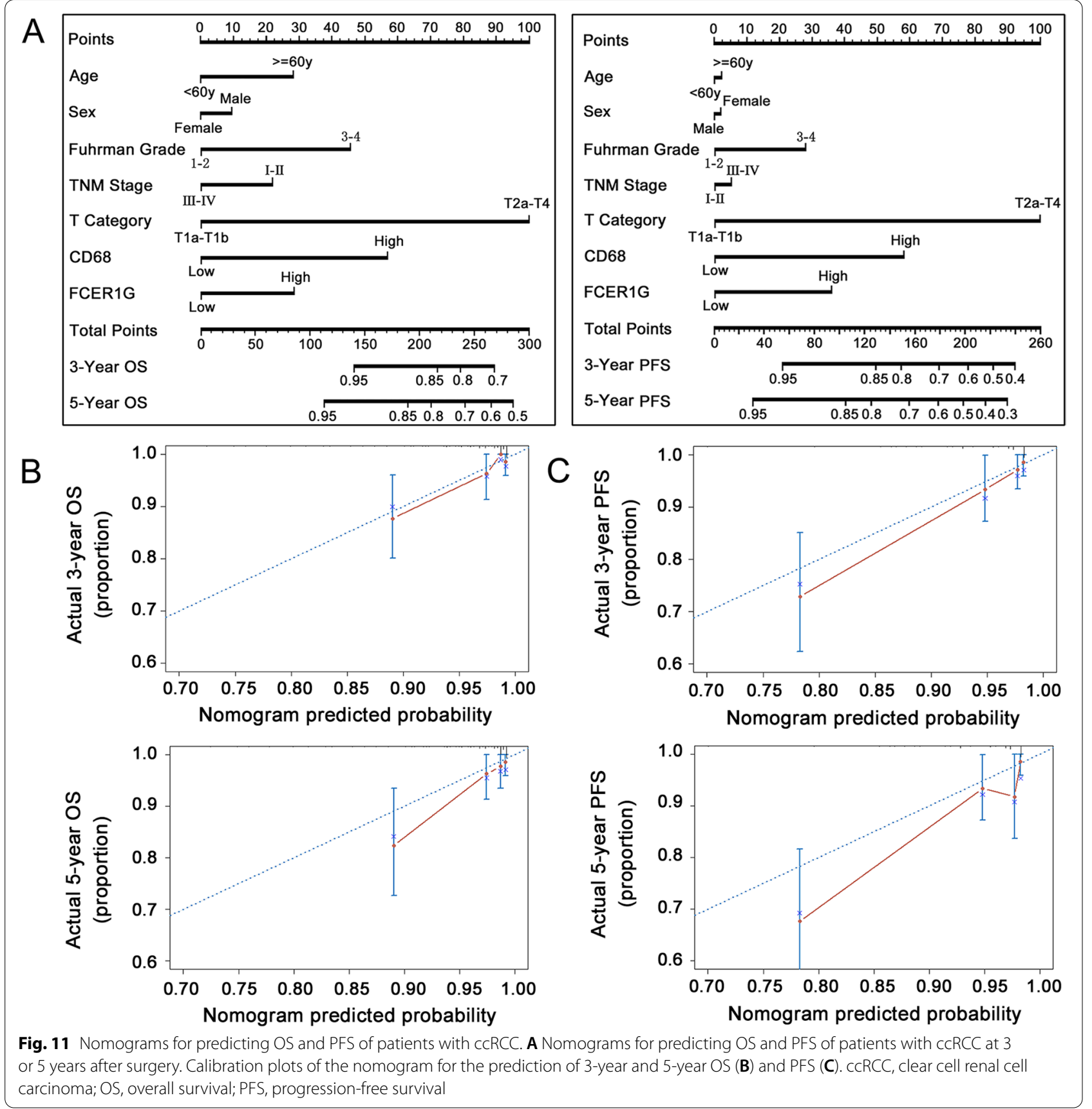

macrophages in ccRCC by the increased secretion of IL4. Furthermore, M2 macrophages derive their tumorsuppressive function partly by suppressing cytotoxic $\mathrm{T}$ cells. This may explain the correlation of FCER1G with both macrophages and $\mathrm{T}$ cell function.

The prognostic role of FCER1G expression in ccRCC has been previously discovered through proteinprotein interaction networks [40] or weighted gene co-expression network [41] analysis of DEGs using open-source bulk RNA-sequencing data of ccRCC.
Another study used the pre-established ESTMATE algorithm [42] to assess the genes with prognostic value in the immune microenvironment of ccRCC, identifying FCER1G as a candidate gene [43]. Nevertheless, there are no studies conducted so far that investigated the correlation of FECER1G expression with macrophages or validated theirfindings in a large clinical cohort. Although the GSEA analysis of high FECER1G expression in TCGA-KIRC was performed by Wang and his colleague, they failed to fully interpret the 
function of FCER1G in suppressing T cells in ccRCC [40]. Unlike previously studies, we identified FCER1G as prognosis marker of ccRCC based on its correlation with macrophage markers.

This study was characterized by certain limitations. Firstly, the relationship of FCER1G expression with macrophage infiltration and suppressed $\mathrm{T}$ cell function are simply concluded by the correlation analysis. Further experiments are required to elucidate the exact cell type in which FCER1G is overexpressed. This is urgently needed, as it may provide insight into the underlying mechanism of FCER1G expression interaction with tumor immunity and affect the prognosis of ccRCC. Secondly, the patient samples included in the study were collected in a single clinical center mainly from 2016 to 2018; most of those patients in TMA-2020 did not reach a 5-year follow-up (range: 33-62 months, median followup: 42 months). Moreover, the death rate in TMA-2020 was only $5.30 \%$, impacting the statistical evaluation of several analyses, such as the multivariate Cox regression. We also observed high FCER1G and CD68 expression levels in patients lost to follow-up, indicating that the death rate in the high FCER1G and CD68 expression group may be underestimated. Multi-centered studies involving large cohorts of patients with ccRCC and a long term follow-up may lead to more accurate conclusions.

\section{Conclusions}

In this study, we used TCGA-KIRC database to investigate genes correlated with macrophages markers and elucidate the association between FCER1G expression and tumor-infiltrating macrophages. FCER1G was abundantly expressed in tumor tissues and showed prognostic importance in patients with ccRCC treated with nivolumab, but not in those treated with everolimus. Combination of FCER1G and macrophage biomarker $C D 68$ can result in better prognostic stratification of patients with ccRCC from TCGA-KIRC. Furthermore, we performed GSEA and predicted the $\mathrm{T}$ cell-suppressive role of FCER1G in ccRCC. We also validated that FCER1G and CD68 are both highly expressed in tumor tissue and correlated with each other. Higher expression of CD68 or FCER1G in ccRCC tissue indicated shorter OS and PFS, and patients with high expression of both CD68 and FCER1G had the worst outcome. Integration of FCER1G and CD68 expression into TNM staging exhibited high accuracy in assessing patients with different prognoses following surgery for ccRCC.

\section{Abbreviations}

TAMs: Tumor-associated macrophages; ccRCC: clear cell renal cell carcinoma; RCC: renal cell carcinoma; TKIs: tyrosine kinase inhibitors; ICl: immune checkpoint inhibitor; RNA-seq: RNA sequencing; DEGs: differentially expressed genes; TMA: tissue microarray; NATs: normal tissues adjacent to tumor;TCGAKIRC: The Cancer Genome Atlas-kidney renal clear cell carcinoma; GSEA: gene set enrichment analysis.

\section{Supplementary Information}

The online version contains supplementary material available at https://doi. org/10.1186/s12885-022-09251-7.

Additional file 1. Detail information of TMA-30.

Additional file 2. Detail information of TMA-2020 NO.1-8.

Additional file 3. Differentially expressed genes correlated with macrophage markers.

Additional file 4. Gene sets enriched in TCGA-KIRC with high FCER1G expression.

\section{Acknowledgements}

The authors would like to acknowledge the Urinary Surgery Department and Pathology Department of Shanghai Changhai hospital for sharing clinical samples.

\section{Authors' contributions}

F.Y. and X.C. conceived and designed the study. K.D. and Wenjin C. performed the majority of the experiments and bioinformatic analysis. X.P. and C.W. performed experiments and analyzed data. Y.S. and C.Q. collected samples. H.W. and Weijie C. followed up the patients via telephone interviews. K.D. and X.P. drafted the manuscript. F.Y. and X.C. reviewed the data and finalized the manuscript. All authors read and approved the final manuscript.

\section{Funding}

This work was supported by the Top-level Clinical Discipline Project of Shanghai Pudong (grant number PWYgf2018-03); the National Natural Science Foundation of China (grant numbers 81772747, 81974391, and 82072806); the Program of Shanghai Academic/Technology Research Leader (grant number 19XD1405100); the Clinical Research Plan of SHDC (grant number SHDC2020CR4025); the Shanghai "Rising Stars of Medical Talent"Youth Development Program: Outstanding Youth Medical Talents (grant to X. Cui) and Youth Medical Talents - Specialist Program (grant to X. Pan); the Shanghai Key Medical Specialties Project (grant number ZK2019A09); the Shanghai Municipal Commission of Health and Family Planning (grant number 20204Y0042); and the Technology Project of Jiading District Health System (grant number 2019-QN-03);National Natural Science Foundation of China (No. 81773154); Shanghai Natural Science Foundation (20ZR1449600), Pudong New Area Science and technology development fund special fund for people's livelihood Research (medical and health) (PKJ2019-Y19)

\section{Availability of data and materials}

The datasets used or analyzed during the current study are available from the corresponding author on reasonable request. The RNA sequencing count data of TCGA-KIRC were downloaded using the R package 'TCGAbiolinks'. Bulk RNA sequencing data of the CheckMate 025 clinical trial are available in the European Genome-Phenome Archive (https://ega-archive.org/): EGAS00001004290, EGAS00001004291, EGAS00001004292.

\section{Declarations}

\section{Ethics approval and consent to participate}

All methods were performed in accordance with the Declaration of Helsinki and relevant regulations. Ethical and operational approval of all research procedures was provided by the Scientific Research Review and Investigation Committee of the Third Affiliated Hospital of the Second Military Medical University (ID:EHBHKY2020-K-026, approval date: August $17^{\text {th }}, 2020$ ), and written informed consent was provided by all patients.

\section{Consent for publication}

NOT APPLICABLE 


\section{Competing interests}

The authors declare that they have no competing interests

\section{Author details}

${ }^{1}$ Department of Urinary Surgery, Third Affiliated Hospital of Naval Medical University (Eastern Hepatobiliary Surgery Hospital), 700 North Moyu Road, 201805 Shanghai, China. ${ }^{2}$ Post-graduate Training Base in Shanghai Gongli Hospital, Post-graduate College, Ningxia Medical University, Yinchuan, China. ${ }^{3}$ Department of Urinary Surgery, Gongli Hospital, Second Military Medical University (Naval Medical University), 219 Miaopu Road, 200135 Shanghai, China. ${ }^{4}$ The Department of Medical Genetics, Naval Medical University, 800 Xiangyin Road, 200433 Shanghai, China.

\section{Received: 28 June 2021 Accepted: 28 January 2022} Published online: 04 February 2022

\section{References}

1. Hsieh JJ, Purdue MP, Signoretti S, Swanton C, Albiges L, Schmidinger M, et al. Renal Cell Carcinoma. Nat Rev Dis Primers. 2017;3:17009. doi: https:// doi.org/10.1038/nrdp.2017.9

2. Lalani AA, McGregor BA, Albiges L, Choueiri TK, Motzer R, Powles T, et al. Systemic Treatment of Metastatic Clear Cell Renal Cell Carcinoma in 2018: Current Paradigms, Use of Immunotherapy, and Future Directions. Eur Urol. 2019;75(1):100-10. doi: https://doi.org/10.1016/j.eururo. 2018.10.010

3. Choueiri TK, Escudier B, Powles T, Mainwaring PN, Rini Bl, Donskov F, et al. Cabozantinib versus Everolimus in Advanced Renal-cell Carcinoma. N Engl J Med. 2015;373(19):1814-23. doi: https://doi.org/10.1056/NEJMo a1510016

4. Díaz-Montero CM, Rini BI, Finke JH. The Immunology of Renal Cell Carcinoma. Nat Rev Nephrol. 2020;16(12):721-35. doi: https://doi.org/10.1038/ s41581-020-0316-3

5. Motzer RJ, Tannir NM, McDermott DF, Arén Frontera O, Melichar B, Choueiri TK, et al. Nivolumab plus Ipilimumab versus Sunitinib in Advanced Renal-cell Carcinoma. N Engl J Med. 2018;378(14):1277-1290. doi: https:// doi.org/10.1056/NEJMoa1712126

6. Choueiri TK, Powles T, Burotto M, Escudier B, Bourlon MT, Zurawski B, et al. Nivolumab plus Cabozantinib versus Sunitinib for Advanced Renal-cell Carcinoma. N Engl J Med. 2021;384(9):829-841. doi: https://doi.org/10. 1056/NEJMoa2026982

7. Rini Bl, Plimack ER, Stus V, Gafanov R, Hawkins R, Nosov D, et al. Pembrolizumab plus Axitinib versus Sunitinib for Advanced Renal-cell Carcinoma. N Engl J Med. 2019;380(12):1116-27. doi: https://doi.org/10.1056/NEJMo a1816714

8. Rini Bl, Powles T, Atkins MB, Escudier B, McDermott DF, Suarez C, et al. Atezolizumab plus Bevacizumab versus Sunitinib in Patients with Previously Untreated Metastatic Renal Cell Carcinoma (IMmotion151): A Multicentre, Open-label, Phase 3, Randomised Controlled Trial. Lancet. 2019;393(10189):2404-15. doi: https://doi.org/10.1016/S0140-6736(19) 30723-8

9. Albiges L, Tannir NM, Burotto M, McDermott D, Plimack ER, Barthélémy P, et al. Nivolumab plus I pilimumab versus Sunitinib for First-line Treatment of Advanced Renal Cell Carcinoma: Extended 4-year Follow-up of the Phase III CheckMate 214 Trial. ESMO Open. 2020;5(6):e001079. doi: https://doi.org/10.1136/esmoopen-2020-001079

10. Jonasch E, Walker CL, Rathmell WK. Clear Cell Renal Cell Carcinoma Ontogeny and Mechanisms of Lethality. Nat Rev Nephrol. 2021;17(4):24561. doi: https://doi.org/10.1038/s41581-020-00359-2

11. Chevrier S, Levine JH, Zanotelli VRT, Silina K, Schulz D, Bacac M, et al. An Immune Atlas of Clear Cell Renal Cell Carcinoma. Cell. 2017;169(4):736 49.e18. doi: https://doi.org/10.1016/j.cell.2017.04.016

12. Toge H, Inagaki T, Kojimoto Y, Shinka T, Hara I. Angiogenesis in Renal Cel Carcinoma: the Role of Tumor-associated Macrophages. Int J Urol. 2009; 16(10):801-7. doi: https://doi.org/10.1111/j.1442-2042.2009.02377.x

13. Qian BZ, Pollard JW. Macrophage Diversity Enhances Tumor Progression and Metastasis. Cell. 2010;141(1):39-51. doi: https://doi.org/10.1016/j.cell. 2010.03.014

14. Dannenmann SR, Thielicke J, Stöckli M, Matter C, von Boehmer L, Cecconi $V$, et al. Tumor-associated Macrophages Subvert T-cell Function and
Correlate with Reduced Survival in Clear Cell Renal Cell Carcinoma. Oncoimmunology. 2013;2(3):e23562. doi: https://doi.org/10.4161/onci.23562

15. Wang $C$, Wang Y, Hong T, Ye J, Chu C, Zuo L, et al. Targeting a Positive Regulatory Loop in the Tumor-macrophage Interaction Impairs the Progression of Clear Cell Renal Cell Carcinoma. Cell Death Differ. 2021;28(3):932-51. doi: https://doi.org/10.1038/s41418-020-00626-6

16. Wang C, Wang Y, Hong T, Cheng B, Gan S, Chen L, et al. Blocking the Autocrine Regulatory Loop of Gankyrin/STAT3/CCL24/CCR3 Impairs the Progression and Pazopanib Resistance of Clear Cell Renal Cell Carcinoma. Cell Death Dis. 2020;11(2):117. doi: https://doi.org/10.1038/ s41419-020-2306-6

17. Cassetta L, Pollard JW. Targeting Macrophages: Therapeutic Approaches in Cancer. Nat Rev Drug Discov. 2018;17(12):887-904. doi: https://doi.org/ $10.1038 /$ nrd.2018.169

18. Colaprico A, Silva TC, Olsen C, Garofano L, Cava C, Garolini D, et al. TCGAbiolinks: an R/Bioconductor Package for Integrative Analysis of TCGA Data. Nucleic Acids Res. 2016;44(8):e71. doi: https://doi.org/10.1093/nar/ gkv1507

19. Risso D, Schwartz K, Sherlock G, Dudoit S. GC-content Normalization for RNA-Seq Data. BMC Bioinformatics. 2011;12:480. doi: https://doi.org/10. 1186/1471-2105-12-480

20. Braun DA, Hou Y, Bakouny Z, Ficial M, Sant'Angelo M, Forman J, et al. Interplay of Somatic Alterations and Immune Infiltration Modulates Response to PD-1 Blockade in Advanced Clear Cell Renal Cell Carcinoma. Nat Med. 2020;26(6):909-18. doi: https://doi.org/10.1038/ s41591-020-0839-y

21. Yu G, Wang LG, Han Y, He QY. clusterProfiler: an R Package for Comparing Biological Themes among Gene Clusters. OMICS. 2012;16(5):284-7. doi: https://doi.org/10.1089/omi.2011.0118

22. Wynn TA, Chawla A, Pollard JW. Macrophage Biology in Development, Homeostasis and Disease. Nature. 2013:496(7446):445-55. doi: https:// doi.org/10.1038/nature12034

23. Gordon S, Taylor PR. Monocyte and Macrophage Heterogeneity. Nat Rev Immunol. 2005;5(12):953-64. doi: https://doi.org/10.1038/nri1733

24. Schelker M, Feau S, Du J, Ranu N, Klipp E, MacBeath G, et al. Estimation of immune cell content in tumour tissue using single-cell RNA-seq data. Nat Commun. 2017;8(1):2032. https://doi.org/10.1038/s41467-017-02289-3.

25. Zhang X, Lan Y, Xu J, Quan F, Zhao E, Deng C, et al. Cell Marker: a manually curated resource of cell markers in human and mouse. Nucleic Acids Res. 2019;47(D1):D721-8. https://doi.org/10.1093/nar/gky900.

26. LiT, Fu J, Zeng Z, Cohen D, Li J, Chen Q, Li B, Liu XS. TIMER2.0 for analysis of tumor-infiltrating immune cells. Nucleic Acids Res. 2020:48(W1):W509W514. doi: https://doi.org/10.1093/nar/gkaa407.

27. Zhu C, Anderson AC, Schubart A, Xiong H, Imitola J, Khoury SJ, et al. The Tim-3 Ligand Galectin-9 Negatively Regulates T Helper Type 1 Immunity. Nat Immunol. 2005;6(12):1245-52. doi: https://doi.org/10.1038/ni1271

28. Kogure A, Shiratori I, Wang J, Lanier LL, Arase H. PANP is a Novel O-glycosylated PILRa Ligand Expressed in Neural Tissues. Biochem Biophys Res Commun. 2011:405(3):428-33. doi: https://doi.org/10.1016/j.bbrc.2011.01.047

29. Zhao Y, Wu X, Li X, Jiang LL, Gui X, Liu Y, et al. TREM2 is a Receptor for $\beta$-Amyloid that Mediates Microglial Function. Neuron. 2018;97(5):10231031.e7. doi: https://doi.org/10.1016/j.neuron.2018.01.031

30. Horstick EJ, Linsley JW, Dowling Jل Jauser MA, MCDonald KK, AshleyKoch A et al. Stac3 is a Component of the Excitation-Contraction Coupling Machinery and Mutated in Native American Myopathy. Nat Commun. 2013;4:1952. doi: https://doi.org/10.1038/ncomms2952

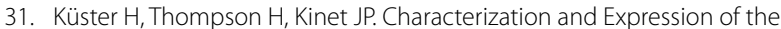
Gene for the Human Fc Receptor Gamma Subunit. Definition of a New Gene Family. J Biol Chem. 1990;265(11):6448-52.

32. Fu L, Cheng Z, Dong F, Quan L, Cui L, Liu Y, et al. Enhanced Expression of FCER1G Predicts Positive Prognosis in Multiple Myeloma. J Cancer. 2020:11(5):1182-94. doi: https://doi.org/10.7150/jca.37313

33. Amo G, Cornejo-García JA, García-Menaya JM, Cordobes C, Torres MJ, Esquevillas G, et al. FCERI and Histamine Metabolism Gene Variability in Selective Responders to NSAIDS. Front Pharmacol. 2016;7:353. doi: https://doi.org/10.3389/fphar.2016.00353

34. Duhan V, Hamdan TA, Xu HC, Shinde P, Bhat H, Li F, et al. NK Cell-intrinsic FceRly Limits CD8+ T-cell Expansion and thereby Turns an Acute into a Chronic Viral Infection. PLoS Pathog. 2019;15(6):e1007797

35. Sweet RA, Nickerson KM, Cullen JL, Wang Y, Shlomchik MJ. B Cell-Extrinsic Myd88 and Fcer1g Negatively Regulate Autoreactive and Normal B Cell 
Immune Responses. J Immunol. 2017;199(3):885-93. doi: https://doi.org/ 10.4049/jimmunol.1600861

36. Brandsma AM, Hogarth PM, Nimmerjahn F, Leusen JH. Clarifying the Confusion between Cytokine and Fc Receptor "Common Gamma Chain". Immunity. 2016;45(2):225-6. doi: https://doi.org/10.1016/j.immuni.2016. 07.006

37. Naqvi AR, Fordham JB, Nares S. MicroRNA target Fc receptors to regulate Ab-dependent Ag uptake in primary macrophages and dendritic cells. Innate Immun. 2016;22(7):510-21. https://doi.org/10.1177/1753425916 661042.

38. Rumsaeng V, Vliagoftis H, Oh CK, Metcalfe DD. Lymphotactin Gene Expression in Mast Cells Following Fc(epsilon) Receptor I Aggregation: Modulation by TGF-beta, IL-4, Dexamethasone, and Cyclosporin A. J Immunol. 1997;158(3):1353-60.

39. Zhu L, Zhao Q, Yang T, Ding W, Zhao Y. Cellular Metabolism and Macrophage Functional Polarization. Int Rev Immunol. 2015;34(1):82-100. doi: https://doi.org/10.3109/08830185.2014.969421

40. Wang L, Lin Y, Yuan Y, Liu F, Sun K. Identification of TYROBP and FCER1G as Key Genes with Prognostic Value in Clear Cell Renal Cell Carcinoma by Bioinformatics Analysis. Biochem Genet. 2021. doi: https://doi.org/10. 1007/s10528-021-10061-y.

41. Chen L, Yuan L, Wang Y, Wang G, Zhu Y, Cao R, et al. Co-expression Network Analysis Identified FCER1G in Association with Progression and Prognosis in Human Clear Cell Renal Cell Carcinoma. Int J Biol Sci. 2017;13(11):1361-72. doi: https://doi.org/10.7150/ijbs.21657

42. Yoshihara K, Shahmoradgoli M, Martínez E, Vegesna R, Kim H, TorresGarcia W, et al. Inferring Tumour Purity and Stromal and Immune Cell Admixture from Expression Data. Nat Commun. 2013;4:2612. doi: https:// doi.org/10.1038/ncomms3612

43. Yin X, Zhang X, Liu Z, Sun G, Zhu X, Zhang H, et al. Assessment for Prognostic Value of Differentially Expressed Genes in Immune Microenvironment of Clear Cell Renal Cell Carcinoma. Am J Transl Res. 2020;12(9):5416-32

\section{Publisher's Note}

Springer Nature remains neutral with regard to jurisdictional claims in published maps and institutional affiliations.

- fast, convenient online submission

- thorough peer review by experienced researchers in your field

- rapid publication on acceptance

- support for research data, including large and complex data types

- gold Open Access which fosters wider collaboration and increased citations

- maximum visibility for your research: over $100 \mathrm{M}$ website views per year

At BMC, research is always in progress.

Learn more biomedcentral.com/submissions 